\title{
Periodic self-modulation of an electrodynamically driven heated wire near resonance
}

\author{
Guillaume Penelet $^{\mathrm{a})}$ and Steven L. Garrett \\ Laboratoire d' Acoustique de l'Université du Mans, Unité Mixte de Recherche, Centre National de la \\ Recherche Scientifique 6613, Avenue Olivier Messiaen, 72085 Le Mans Cedex 9, France
}

(Received 2 August 2018; revised 31 January 2019; accepted 31 January 2019; published online 22 February 2019)

\begin{abstract}
A thin nichrome wire driven near resonance by the Lorentz force and heated by an alternating electrical current is a popular lecture demonstration. Due to the convective cooling of the portions of the wire moving with the greatest amplitude, only glowing regions near a velocity node will be visible in a darkened room. Nonlinear effects and the thermal expansion coefficient of the wire displace the wire's tensioning mass. By adiabatic invariance, the work done on or by the vibrating wire, due to the changes in the mass's elevation, causes the natural frequency of the standing wave resonance to be shifted. Competition between the thermal inertia of the wire and the convective heat transfer coefficient introduces an exponential thermal relaxation time so that the amplitude of vibration is dependent on the ratio of the drive frequency to the changing resonance frequency at an earlier (retarded) time. These thermal and kinetic effects are incorporated into three coupled nonlinear ordinary differential equations that are separated by the method of multiple time scales and are solved numerically, reproducing both the spontaneous appearance of stable periodic amplitude modulation and the hysteretic behavior observed with increasing or decreasing of the drive frequency. (C) 2019 Acoustical Society of America. https://doi.org/10.1121/1.5091094
\end{abstract}

[JFL]

Pages: 998-1017

\section{INTRODUCTION}

\section{A. A popular lecture demonstration}

Physics instructors teaching introductory courses will often demonstrate standing waves on strings by exciting a string that is attached at one end to a vibrator (e.g., a small electrodynamic shaker or a suitably modified electrodynamic loudspeaker) and is tensioned by passing the other end of the string (usually a cotton or synthetic cord) over a pulley that acts as a second rigid termination and allows a mass, $M$, hung from the string, to provide a uniform tension, $\mathcal{T}=M g$, where $g$ is the local gravitational acceleration. Along with this standard apparatus, a strobe light is frequently used to examine the resulting "loops and nodes" in slow motion when the driven frequency, $f$, is an integer multiple of the fundamental standing wave frequency, $f_{1}$,

$$
f_{1}=\frac{c_{t}}{2 L}=\frac{1}{2 L} \sqrt{\frac{\mathcal{T}}{\rho_{L}}} .
$$

The distance between the vibrating support and the pulley is $L$ (i.e., the string's "speaking length") and the propagation speed of transverse vibrations is $c_{t}$. For our vibrating nichrome wire, this transverse wave speed is typically less than $100 \mathrm{~m} / \mathrm{s}$ whereas the speed of compressional waves in the wire is greater than $5000 \mathrm{~m} / \mathrm{s}$. The linear mass density of the string or wire, $\rho_{L}$, is taken to be approximately constant, since the string's temperature is constant, and is equal to the ratio of the string's mass, $m_{s}$, between the fixed ends, and the

\footnotetext{
${ }^{\text {a)} E l e c t r o n i c ~ m a i l: ~ g u i l l a u m e . p e n e l e t @ u n i v-l e m a n s . f r ~}$
}

separation between those ends, $L: \rho_{L}=m_{s} / L$. This resonance condition corresponds to placing an integer number, $n$, of half-wavelengths between those rigid boundaries. In a linear analysis for infinitesimal amplitude vibration, both $\rho_{L}$ and $\mathcal{T}$ are considered to be constant. However, for a very "light" string under a very "weak" tension the equations of motion for the string would be nonlinear.

The mechanical output impedance of the electrodynamic driver used in such lecture demonstrations is typically much greater than the mechanical input impedance that the string can present to the driver, so the driver's amplitude is not influenced by the vibrations of the attached string, thus the driver acts as the source of a constant displacement amplitude excitation. The resonance condition for the linear system without damping, as expressed in Eq. (1), is therefore a consequence of the fact that the driver can impose a nonzero transverse displacement on the string at the end where it also supplies the nominally "fixed" boundary condition. This resonance condition is commonly explained by pointing out that the amplitude of the string will be everywhere infinite if a non-zero transverse displacement is imposed at a location in the standing wave that would otherwise be a node. However, dissipation and nonlinear effects conspire to limit the steady-state amplitude of the resonance response. ${ }^{1}$

In more advanced courses on acoustics and vibration, a more elaborate but similar lecture demonstration is used to show that if the wire is driven by a constant amplitude localized force that varies sinusoidally in time, then that force will produce the greatest resonance response if it is applied at a displacement anti-node. ${ }^{2}$ A popular version of this lecture demonstration involves an electrically heated wire that glows bright red-orange in a darkened lecture room and 
is known as the "burning wire." The disappearance of the vibrating anti-nodal portions of the wire is due to convective cooling, while the regions adjacent to a node still glow in a darkened room from the heating. This is shown in photos that appear in an article by Gluck. ${ }^{3}$ In Gluck's demonstration, the current carrying wire was vibrated by an electrodynamic shaker, not by the interaction of an oscillating current through the wire with a permanent magnet.

This paper will focus on such a burning wire demonstration, but an alternating electrical current is passed through our wire and a small "horseshoe magnet" is located near the center of the wire as shown in Fig. 1(a). The combination of the alternating current of frequency, $f$, and the magnetic field, $\vec{B}$, creates a transverse Lorentz force, $\vec{F}_{\perp}=(\overrightarrow{i w}) \times \vec{B}$, where $w$ is the width of the magnet's closely spaced pole pieces surrounding the wire and $\vec{i}$ is the alternating current that is oscillating along the wire's direction. The frequency of that electrical current, $f$, is typically tuned to be close to one of the wire's normal mode frequencies, $f_{n}=n f_{1}$, where $n$ is a non-zero integer, to produce a large resonance response of the wire.

\section{B. Observation of a self-maintained amplitude modulation}

Just a few years ago (circa 2015), when the electrodynamically force-driven version of this demonstration was recreated at the Laboratoire d'Acoustique de l'Université du Mans, a steady modulation of the standing wave's amplitude was observed with modulation periods of about a second.
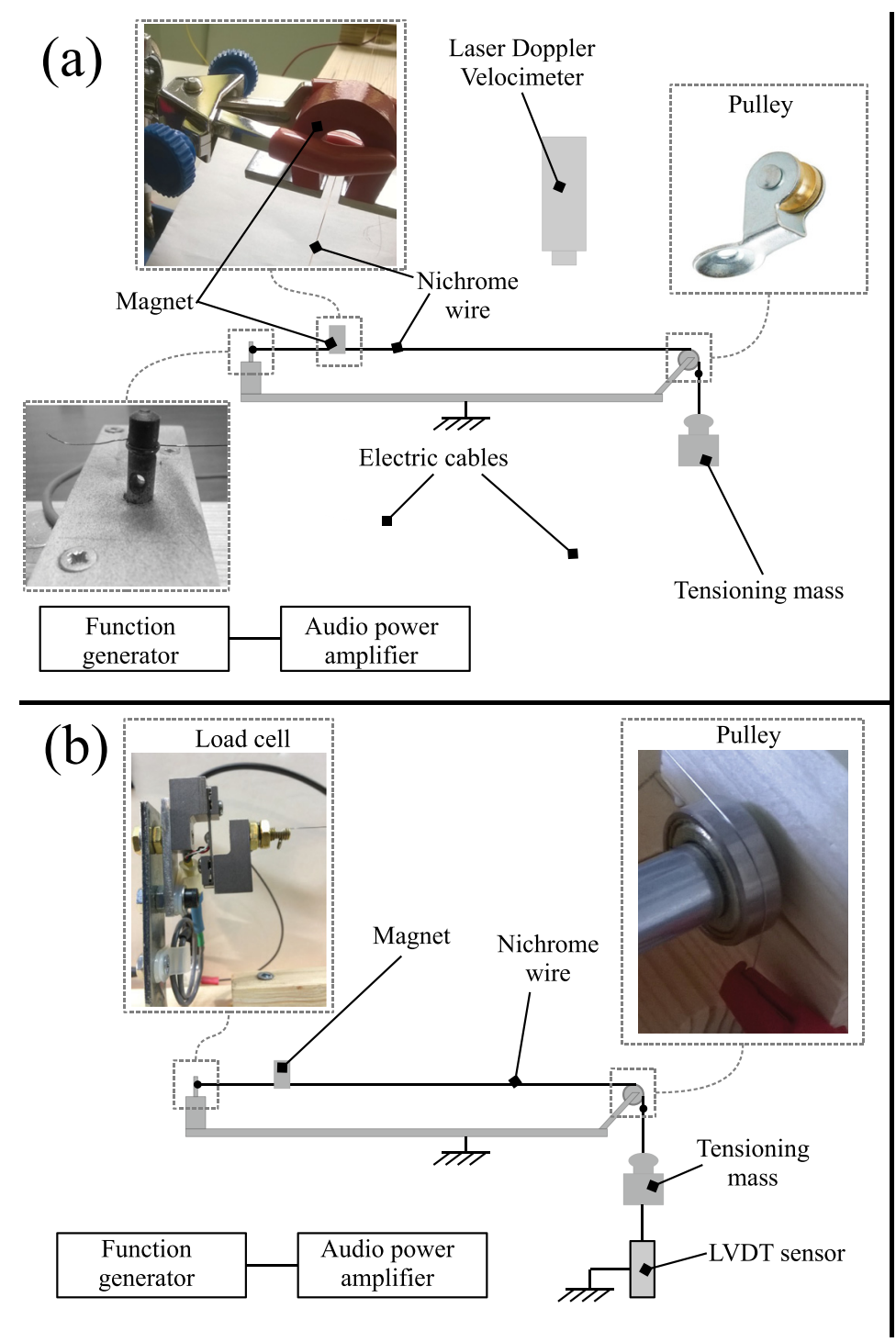

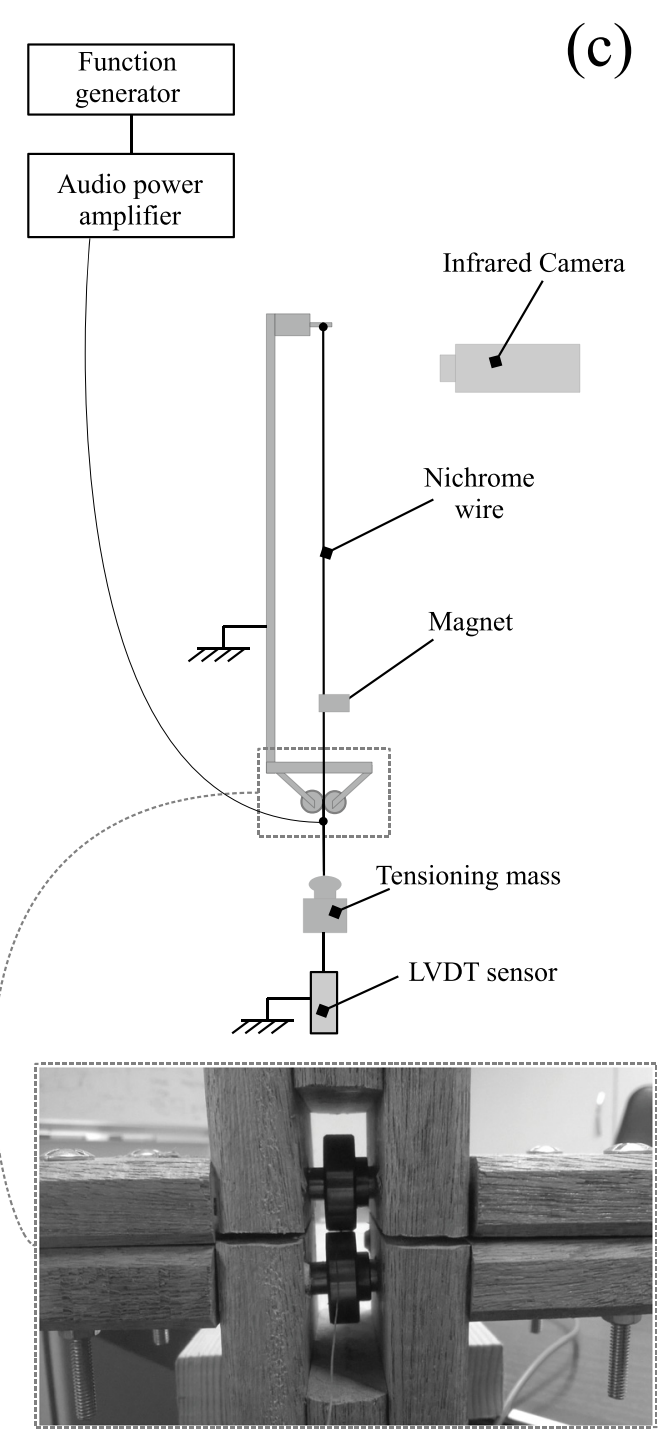

FIG. 1. (Color online) Schematic drawings of the three versions of the apparatus, with photographs of important elements. (a) Sketch of the first apparatus. The end connected to the tensioning mass is equipped with the small "rope" pulley (see photograph). The other end is made with a guitar tuning head. The horseshoe magnet (Ref. 16) and its two pole pieces are supported on a lab jack (see photographs). A laser-Doppler velocimeter is positioned over the wire near its center. (b) Sketch of the second apparatus. The end connected to the tensioning mass is made with a ball-bearing race, and a thin-beam load cell (Ref. 17) provided the rigid termination (see photographs). The NiCr wire is attached to the load cell through a half-inch long, 10-32 threaded brass shaft that has a hole drilled to allow the wire to be passed through. The wire is constrained by a small bead (visible below the brass shaft), in much the same way as the end bead that is ordinarily provided with a guitar string at one end. (c) Sketch of the third apparatus, that was used to make high-speed infrared images of the heated wire like that shown in Fig. 6. There are two ball-bearing races that constrain the transverse motion of the wire at the lower end of the apparatus (see photograph). 
That version of the "burning wire" demonstration used smaller diameter nichrome wire $(\cong 0.25 \mathrm{~mm})$ than had been used in other versions of this lecture demonstration. This paper will explain the mechanisms that conspire to produce this periodic self-modulation of a heated electrodynamically driven wire near its fundamental standing wave resonance frequency.

There are many studies that have been published over more than a century, dating back to Kirchhoff ${ }^{4}$ and Lord Rayleigh, ${ }^{5}$ that have examined large-amplitude effects in vibrating strings, including amplitude modulation, as well as hysteretic behavior, sub-harmonic bifurcations, and transition to chaotic behavior. However, those results were for strings that were not also being heated by the driving current. It is interesting to note that the amplitude modulation, reported by Johnson and Bajaj, ${ }^{6}$ is based on a calculation and occurs theoretically only when the vibration of the wire is circularly polarized, not when the polarization is confined to a single plane. Johnson and Bajaj claim: "The resulting limit cycle solutions correspond to amplitude modulated whirling or ballooning motions of the string."

For the most part, the forced vibrating finite-amplitude (and non-heated) string has been studied as a mathematical problem in the field of nonlinear differential equations. Those earlier models ${ }^{1,7}$ rely on the fact that the mean tension of the wire is increased as the amplitude of the vibration is increased. Such a nonlinear model, based on a mass oscillating perpendicular to the line which joins the mass to the two strings was proposed by Tufillaro who makes the following claim: "In addition to whirling, several other interesting phenomena are easily observed in such forced strings, including periodic and aperiodic cycling between large and small amplitudes."7 Tufillaro did not report any experimental results to compare with his theory.

\section{Qualitative description of the modulation mechanisms}

This paper will report on several experiments and provide both theoretical analyses of the mechanisms responsible for the modulation as well as numerical simulations based on coupled differential equations that incorporate those mechanisms. Before executing such a detailed analysis, it will be helpful to provide the reader a context to understanding the modulation mechanism, using the following description of events which is outlined below:

(1) The wire is driven by an alternating electrical current at a frequency, $f$, that is just below the wire's fundamental natural (resonance) frequency, $f<f_{1}$.

(2) The Joule heating produced by the electrical current raises the wire's temperature, $T$.

(3) Due to the wire's positive coefficient of thermal expansion, the tensioning mass descends, thus causing the natural frequency, $f_{1}(T)$, to decrease in accordance with adiabatic invariance (i.e., the mass does work on the wire). This change in natural frequency brings the natural frequency, $f_{1}(T)$, closer to the drive frequency, $f$, thus increasing the amplitude of the wire's vibration.
(4) The resulting increase in the wire's transverse velocity enhances the convective cooling of the wire by the surrounding (stationary) air.

(5) The wire cools, but does so with a time lag determined by the wire's thermal inertia (i.e., its heat capacity) and the effective thermal resistance between the wire's surface and the air's convectively enhanced heat transfer.

(6) The convective cooling causes the wire to contract and raises the tensioning mass, thus raising the wire's natural frequency, $f_{1}(T)$, again by adiabatic invariance, since now the shrinking wire is doing work on the mass. This change in natural frequency makes the drive frequency, $f$, further below the natural frequency, $f_{1}(T)$, thus decreasing the amplitude of the wire's transverse vibration.

(7) The resulting decrease in the wire's transverse velocity reduces the convective cooling of the wire by the surrounding (stationary) air, heating the wire, and the process repeats by returning to step (3).

\section{Organization}

The details of the previous qualitative description are presented in the remainder of this paper. In Sec. II, three similar experimental set-ups are described that differ primarily in the instrumentation used to study the wire's vibration. Section III reports experimental results on the measured modulation period and the asymmetry and hysteresis of the resonance tuning curve.

Section IV provides a detailed calculation that extends the accepted correlations for the convective cooling of an infinitely long cylinder in a steady flow field to the case of a wire oscillating in a stagnant fluid by application of the Iguchi hypothesis. That analysis leads to an expression for the wire's steady-state temperature as a function of its transverse velocity. The resulting lumped-element thermal resistance of that convective heat transfer process is combined with the wire's thermal inertia to calculate the exponential time constant for relaxation of the wire's temperature. That section concludes with a confirmation of the predicted temperature distribution provided by high-speed infrared images of a stationary and a vibrating wire.

Section V addresses the relationship between the wire's steady-state vibrational amplitude as a function of the tuning ratio, $f / f_{1}(T)$, and the quality factor, $Q_{1}$. The shift in the wire's fundamental natural frequency with temperature, $f_{1}(T)$, is reported and justified by application of the Boltzmann-Ehrenfest principle of adiabatic invariance. An examination of the indices of traditional acoustics textbook $^{1,8-15}$ reveals that adiabatic invariance is not addressed, so a few simple examples of its application to similar problems are also provided in the Appendix.

Having established the relevant parameters that control the amplitude modulation and experimentally determining their values for this apparatus, the qualitative understanding is reiterated in Sec. VI before the wire's vibration is modelled through a set of coupled differential equations in Sec. VII. Also in that section, the amplitude envelop of the transverse vibrations is extracted from those equations by the method of multiple time scales and the resulting set of 
coupled ordinary differential equations are solved numerically with those results presented graphically in Sec. VIII.

\section{EXPERIMENTAL APPARATUS}

During the course of the investigations reported here, three versions of an apparatus for applying tension and electrodynamically exciting a nichrome $(\mathrm{NiCr})$ wire were constructed. They are summarized in Table I and in Fig. 1. All three apparatuses were made using wooden frames and the distance between the fixed end and the pulley that is also used to apply the alternating current was set to $L \cong 52.5 \mathrm{~cm}$. The first two configurations operated horizontally and the third operated vertically. Figure 1 shows a schematic drawing for each version, as well as photographs of some elements like the load cell and the three versions of pulley. The three versions of the apparatus provided a fixed attachment to the $\mathrm{NiCr}$ wire at one end and a block and pulley system at the other end that transferred the gravitational force, $M g$, from the suspended mass to tension the wire. Electrical connections were provided to the fixed end and to the pulley to transmit the alternating electrical current along the wire.

All of these experimental configurations exhibited the periodic amplitude modulation and used \#30-gauge nickelchromium alloy wire $(60 \% \mathrm{Ni}, 16 \% \mathrm{Cr}$, and $24 \% \mathrm{Fe})$ with a nominal diameter of $0.010 \mathrm{in} . \cong 250 \mu \mathrm{m} .{ }^{18}$ The electrical resistance of that wire, $R_{d c}$, between the fixed termination and the pulley was about $12 \Omega$. The temperature coefficient of resistance for the wire was carefully measured to allow subsequent resistance measurements to infer the average temperature of the vibrating wire. Using a stirred bath of heated peanut oil,${ }^{19}$ measurements showed $\left(1 / R_{0}\right)\left(d R_{N i C r} / d T\right)=(1.576 \pm 0.001) \times 10^{-4} /{ }^{\circ} \mathrm{C}$ for $32^{\circ} \mathrm{C}$ $<T_{\text {wire }}<143{ }^{\circ} \mathrm{C}$, where $R_{0}=11.06 \Omega$ at $32.1^{\circ} \mathrm{C}$. This result is consistent with the manufacturer's specification of $0.00015 /{ }^{\circ} \mathrm{C}$ for $20^{\circ} \mathrm{C} \leq T \leq 500{ }^{\circ} \mathrm{C}$.

The second and third versions used a weight "hanger" that was threaded below the weight platform to accept the plunger of a linear variable differential transformer (LVDT) ${ }^{20}$

TABLE I. Summary of the three systems, their sensors, and the results each provided.

\begin{tabular}{lccc}
\hline \hline Apparatus & Orientation & Sensors & Results \\
\hline$\# 1$ & Horizontal & $\begin{array}{c}\text { Laser Doppler } \\
\text { velocimeter } \\
\text { Accelerometer }\end{array}$ & Modulation period \\
& & Tuning curves \\
& & Free-decay rate \\
\#2 & Horizontal & $\begin{array}{c}\text { Thin-beam load } \\
\text { cell } \\
\end{array}$ & Modulation period \\
& & LVDT & Natural frequency shift \\
& & Q-wire electrical & Quality factor \\
& & & Motion of the tensioning mass \\
& & Average wire temperature \\
& & Tigh-speed IR & Temperature distribution \\
\#3 & camera & Modulation period \\
& & LVDT & Motion of the tensioning mass \\
\hline \hline
\end{tabular}

so that the displacement of the mass as a function of the wire's temperature and amplitude of vibration could be measured precisely. ${ }^{21}$

The first version used a laser-Doppler velocimeter ${ }^{22}$ to measure the wire's transverse velocity. The velocimeter's laser beam is perpendicular to the wire and directed to the top of the wire thus ensuring that only vertically planepolarized vibrations would be transduced. An alternative measure of the wire's oscillation amplitude was provided by a small accelerometer that was attached to the post that provided the fixed boundary. That first version used a small "rope" pulley similar to one that might support a clothes line, see Fig. 1(a). During experimentation, some slip-stick behavior was observed when the wire's temperature changed and the mass's position was displaced due to the wire's linear coefficient of thermal expansion, $\alpha_{\mathrm{NiCr}}$.

The second version of the apparatus used a ball-bearing race $^{23}$ shown in Fig. 1(b) to transmit the mass's force to the wire and did not exhibit any observed hysteretic behavior. A grooved brass sleeve surrounded the bearing race to provide contact to the $\mathrm{NiCr}$ wire and keep it centered. A thin-beam load cell, ${ }^{17}$ shown in Fig. 1(b), provided the rigid termination at the end opposite to the ball-bearing block and pulley. The load cell included a quartet of strain gauges that formed a full bridge excited by $\pm 5.00 \mathrm{~V}_{d c}{ }^{21}$ The bridge imbalance was applied to an instrumentation amplifier ${ }^{24}$ with a gain of 10.0 $(+20 \mathrm{~dB})$ that provided the three components of the output signal. The dc component of the bridge output, $V_{\text {out }}$, provided the static tension based on a measured calibration constant, $\left(d V_{\text {out }} / d F\right)=4.76 \pm 0.05 \times 10^{-3} \mathrm{~V} \mathrm{~N}^{-1}$. The load cell's output at the wire's driven frequency, $f$, is proportional to the wire's amplitude of vibration and the output at $2 f$ is proportional to the fluctuating component of the wire's tension.

The third version of the apparatus, shown in Fig. 1(c), used two ball-bearing races that were forced together by two machine screws to control the pressure the rollers applied to the wire. The wire's motion and temperature were imaged by a high-speed infrared camera. ${ }^{25}$

A small hobbyist's magnet, ${ }^{16}$ visible in Fig. 1(a), provided the magnetic field. The magnet was sold with a "keeper." Two of those keepers were used as the magnet's pole pieces. They have a height, $h=2.0 \mathrm{~mm}$ and a width, $w=7.0 \mathrm{~mm}$. The two pole pieces were usually separated by about $6 \mathrm{~mm}$ and the magnitude of the magnetic induction between those pole pieces at the center was $|\vec{B}| \cong 1300$ $\mathrm{G}=0.13 \mathrm{~T}$. The wire's motion was driven electrodynamically by the Lorentz force, $\vec{F}=(\vec{i} w) \times \vec{B}$, applied between the magnet's pole faces, where $i$ is the current in the wire, $|\vec{B}|$ is the magnetic induction provided at the gap's center by the horseshoe magnet, and $w$ is the width of the magnet's pole pieces in the direction of the alternating current.

It is worthwhile to note that although the wire's peak-topeak amplitude often exceeded the height, $h$, of the pole pieces, the reduction in the work done by the Lorentz force on the wire is not diminished by as much as the field is reduced at the extremes of the wire's motion. At resonance, the force and velocity are in phase. When the wire passes through its equilibrium position its velocity is greatest, as is the strength of the magnetic field. In a similar situation that 
used a different magnet that made it easier to measure the decrease in $B$ with displacement from equilibrium, it was found that the delivered power decreased by only $6 \%$ while the magnetic field strength was $30 \%$ lower at the extremes of displacement. $^{26}$

\section{EXPERIMENTAL OBSERVATIONS OF MODULATION}

The typical experiment was run with a tensioning mass, $0.5 \mathrm{~kg}<M<1.5 \mathrm{~kg}$, and with an alternating electrical current, $\quad 0.2 A_{a c}<i_{a c}<1.2 A_{a c}$, and an angular frequency, $\omega=2 \pi f \cong 1000 \mathrm{rad} / \mathrm{s}$. Using a function generator to precisely control the excitation frequency, that frequency was initially set lower than the resonance frequency and was then increased, resulting in a growth in the wire's vibrational amplitude in its fundamental half-wavelength standing wave mode. Such a tuning curve is shown in Fig. 2. That tuning curve with a hysteretic shape exhibits the type of behavior characteristic of a nonlinear oscillator. ${ }^{27}$ The wire's vibrational amplitude increases as the resonance frequency is approached from below, but when the resonance frequency is exceeded, the amplitude drops precipitously. If the frequency is reduced from above the resonance frequency, the amplitude does not start increasing again until the frequency is well below that resonance peak frequency that was achieved when the frequency was increasing. This difference in response when increasing and decreasing frequency is illustrated in the simulation shown in Fig. 15.

If the frequency is increased to within a few $\mathrm{Hz}$ from the peak shown in Fig. 2 and held constant, the amplitude of the wire's vibration will start to modulate. The period of those modulations remains constant, typically at one second (see Fig. 3) but occasionally as long as $8 \mathrm{~s}$ (see Fig. 11), if the drive frequency remains constant. That modulation period is only a weak function of the drive frequency, as shown in Fig. 3. It is important to note that during and prior to modulation the polarization of the wire's motion is strictly observed to be linear polarization, unlike the observed whirling modulations mentioned in the Introduction. ${ }^{6,7}$ If the

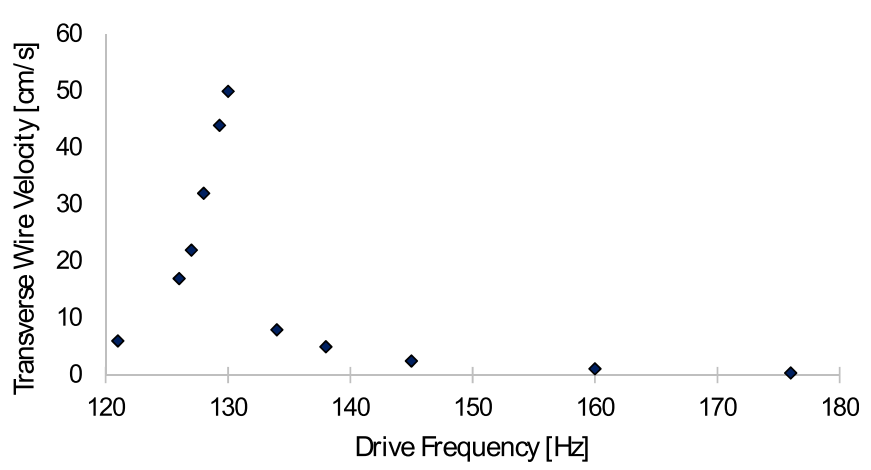

FIG. 2. (Color online) A typical asymmetric tuning curve for the electrodynamically driven heated wire excited by a constant alternating current for increasing frequency. The amplitude of the wire's transverse velocity was measured using the laser-Doppler vibrometer (Ref. 22) that is shown in Fig. 1(a). If the frequency is reduced from above the resonance frequency, the amplitude does not start increasing again until the excitation frequency is well below the peak that was achieved when the frequency was increasing. Such hysteretic behavior is shown in Fig. 15.

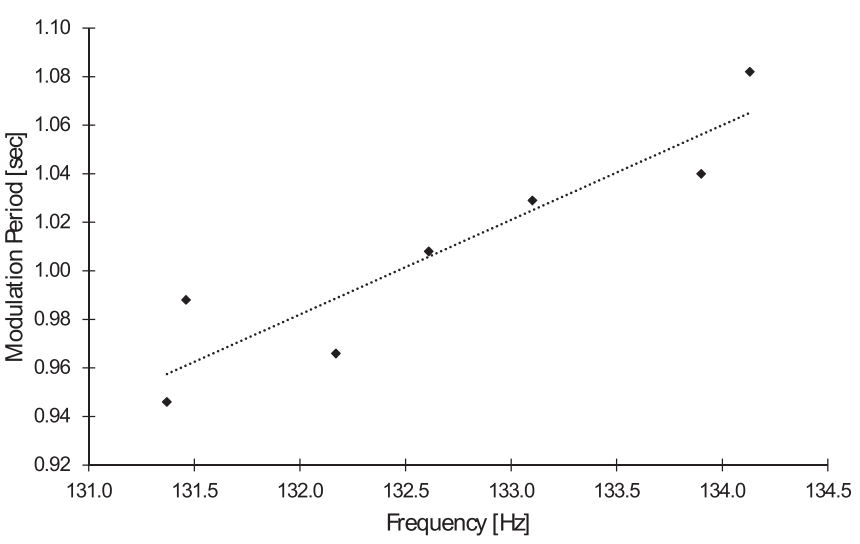

FIG. 3. The modulation period was determined by timing ten modulation cycles with a stopwatch. The dotted line is only intended to show the increasing trend in the modulation period with increases in the drive frequency, $f$. The drive current was held constant at $286 \mathrm{~mA}_{\mathrm{ac}}$. Measurement of the modulation periods could be affected by air currents in the laboratory that would vary due to an open window, people moving in the lab, and the opening or closing of the lab's door.

polarization were not linear, it would not have been possible to measure the wire's velocity using the laser-Doppler velocimeter $^{22}$ shown in Fig. 1(a).

Once the amplitude modulation has been established, it is possible to impose a steady convective air flow by blowing breath over the wire or exposing the wire to the air flow produced by a small electric fan of the type used to cool electronics. $^{28}$ This will cause the vibrations to cease temporarily but the wire will slowly return to its previous state of modulated vibration over a time that is typically less than $10 \mathrm{~s}$.

If the drive frequency is set above the peak in Fig. 2, the vibrational amplitude will be very small. When a steady air flow is again imposed, the wire's vibration amplitude will increase to amplitudes that are the same as when the drive frequency is tuned to produce the maximum response.

These observations led us to conclude that the heating of the wire due to the electrical current flow was essential to the amplitude modulations we observed. To confirm this conclusion, the $250 \mu \mathrm{m}$ diameter $\mathrm{NiCr}$ wire was removed from the first apparatus and was replaced by $303 \mu \mathrm{m}$ diameter aluminum wire and then by a $200 \mu \mathrm{m}$ diameter copper wire. Because the electrical resistances of those materials are far smaller than the electrical resistance of $\mathrm{NiCr}$, the same electrical current flow produces negligible heating. No amplitude modulations were observed with the aluminum wire that had a fundamental half-wavelength resonance frequency, $f_{1}=143 \mathrm{~Hz}$, over a current range $0.2 A_{a c}<i<1.6 A_{a c}$; the vibration amplitude was similar to that of the $\mathrm{NiCr}$ wire, since the electrical currents were similar. The same absence of amplitude modulation was observed with the copper wire that had a fundamental half-wavelength resonance frequency, $f_{1}=112 \mathrm{~Hz}$, over a current range $0.2 A_{a c}<i<1.0 A_{a c}$.

\section{CONVECTIVE COOLING AND EXPERIMENTAL THERMOMETRY}

Being convinced of the importance of the convective cooling of the wire to its amplitude modulation, it became necessary to calculate the magnitude of such convective heat 
transfer mechanisms as a function of the wire's vibrational amplitude. The importance of buoyancy-driven free convective cooling can be evaluated by calculation of the Richardson number, ${ }^{29} \mathrm{Ri}$,

$$
\mathrm{Ri}=\frac{g \beta D(\Delta T)}{v^{2}}=\frac{g D}{v^{2}} \frac{\Delta T}{T_{\infty}} .
$$

For any ideal gas, the thermal expansion coefficient, $\beta=T_{\infty}^{-1}$, where $T_{\infty}$ is the mean absolute $(\mathrm{K})$ temperature of the gas. We will take $T_{\infty}=293 \mathrm{~K}$. For our $\mathrm{NiCr}$ wire, $D=0.25 \mathrm{~mm}$. The resonance angular frequency of the wire's vibration in its fundamental standing wave mode is conveniently close to $1000 \mathrm{rad} / \mathrm{s}$. Typical peak amplitudes for the wire's transverse vibration, $z_{p} \geq 2 \mathrm{~mm}$, so the wire's velocity relative to the stagnant air far from the wire is typically $v \equiv v_{p}=\omega z_{p} \geq 2 \mathrm{~m} / \mathrm{s}$. The temperature difference between the air and the wire can be taken as $\Delta T \cong 50^{\circ} \mathrm{C}$, so substitution of these nominal values into Eq. (2) produces $\mathrm{Ri} \cong 1 \times 10^{-4}$ $\ll 1$, suggesting that buoyancy-driven convection can be ignored. $^{29}$

One attractive feature of the "burning wire" demonstration, which was mentioned in the Introduction, is the fact that the rapidly vibrating sections of the resonating wire do not glow when heated because the enhanced heat transfer, caused by forced convection, cools the portions of the wire that are in rapid motion while the nearly stationary parts of the wire near supports (or near other velocity nodes for modes of higher order) still will glow. ${ }^{2,3}$ Standard treatments of convective cooling use the non-dimensional Nusselt number, $\mathrm{Nu}$, to calculate the convective heat transfer coefficient, $h$, in the presence of a flow velocity, $v$, characterized by a nondimensional Reynolds number, Re,

$$
\operatorname{Re}=\frac{v D \rho}{\mu}=\frac{v D}{\nu} \quad \text { and } \quad \mathrm{Nu}=\frac{h D}{\kappa} .
$$

The fluid density is $\rho$, its shear viscosity is $\mu$, and the kinematic viscosity is $\nu=\mu / \rho . \kappa$ is the thermal conductivity of the fluid. For air at $p_{m}=1 \mathrm{bar} \equiv 100 \mathrm{kPa}$ and at $T_{\infty}=293 \mathrm{~K}$ $\cong 20^{\circ} \mathrm{C}, \mu=1.525 \times 10^{-5} \mathrm{~m}^{2} / \mathrm{s}$, and $\kappa=2.57 \times 10^{-2} \mathrm{~W} /$ $\mathrm{m} / \mathrm{K}$. Substituting the same typical operational conditions used to evaluate the Richardson number in Eq. (2) into Eq. (3), $\operatorname{Re} \cong 33$.

One correlation between $\mathrm{Nu}$ and $\mathrm{Re}$ is provided for steady flow around cylinders of infinite length, ${ }^{30}$

$$
\mathrm{Nu}=C \operatorname{Re}^{m} \operatorname{Pr}^{1 / 3} \text {. }
$$

According to Table II and to our typical operational parameters (i.e., $\operatorname{Re} \cong 33$ ), we will let $C=0.911$ and $m=0.385$. The

TABLE II. Suggested values (Ref. 30) for $C$ and $m$ in Eq. (4) as a function of Re.

\begin{tabular}{lcc}
\hline \hline $\operatorname{Re}$ & $C$ & $m$ \\
\hline $0.4-4$ & 0.989 & 0.330 \\
$4-40$ & 0.911 & 0.385 \\
$40-4000$ & 0.683 & 0.466 \\
\hline \hline
\end{tabular}

Prandtl number of air at mean pressure, $p_{m}=1 \mathrm{bar}$ $=100 \mathrm{kPa}$, and at $T_{\infty}=293 \mathrm{~K}=20^{\circ} \mathrm{C}$ is $\operatorname{Pr}_{\text {air }}=0.709$. For $\mathrm{Re}=33, \mathrm{Nu}=3.12$.

It is common to invoke the "Iguchi hypothesis," for the evaluation of convective effects in oscillatory flows. ${ }^{31}$ This approach assumes that the oscillating system behaves as though it has achieved the steady-state flow configuration at every instant so the average response is then obtained by integrating the motion over a complete cycle.

When the oscillating frequency is fixed close to resonance, the string's displacement, $z(x, t)$, can be written as a harmonic function of both time and space, i.e., $z(x, t)$ $=z_{p} \sin (\omega t) \sin (\pi x / L)$. The time-averaged convective heat transfer coefficient, $\bar{h}$, is the result of an integration of Eq. (4) over one full cycle (or over half a cycle, since the sign of the oscillating velocity does not impact the heat exchange). As illustrated in Fig. 4, the average of $\sin ^{0.385}(\omega t)$ for $0<\omega t<\pi$ will be about 0.8 , so that $\bar{h} \cong 4 h / 5$, where $h$ is estimated using Eq. (4) with the peak velocity $v_{p}$ at the wire's midpoint for the fundamental mode. The same conclusion holds for the average over the string's length $L$, denoted as $\langle h\rangle$, which will be $80 \%$ of the steady flow result, $h$, that relates the maximum displacement amplitude of the wire (at the wire's midpoint for the fundamental mode), $z_{p}$, corresponding to a maximum transverse velocity, $v_{p}=\omega z_{p}$.

Moreover, the shape of the function, $\sin ^{0.385}(\pi x / L)$ for $0<x<L$ shown in Fig. 4, is encouraging since the heat transfer coefficient is at least $50 \%$ of its maximum value over $89 \%$ of the wire's length, thus almost the entire wire disappears, except for the glowing regions close to the velocity nodes, for the lecture demonstration version of this experiment mentioned in the Introduction, ${ }^{2}$

$$
\langle\bar{h}\rangle=\frac{4 \bar{h}}{5}=\frac{4}{5} \frac{\kappa \overline{\mathrm{Nu}}}{D} \cong 250 \mathrm{~W} \mathrm{~m}^{-2} \mathrm{~K}^{-1} .
$$

The calculated result in Eq. (5) corresponds to the same nominal conditions used to evaluate Eqs. (2), (3), and (4). An effective convective heat transfer coefficient, $h_{\mathrm{eff}}$, for the wire can be calculated from Eq. (5) using the wire's surface area, $\pi D L \cong 4.2 \times 10^{-4} \mathrm{~m}^{2}$,

$$
h_{\mathrm{eff}}=\frac{1}{R_{t h}}=\pi D L\langle\bar{h}\rangle \cong 0.106 \mathrm{~W} \mathrm{~K}^{-1} .
$$

As mentioned in Sec. IC that presented a qualitative description of the modulation mechanism, an important feature of the processes which creates the periodic amplitude

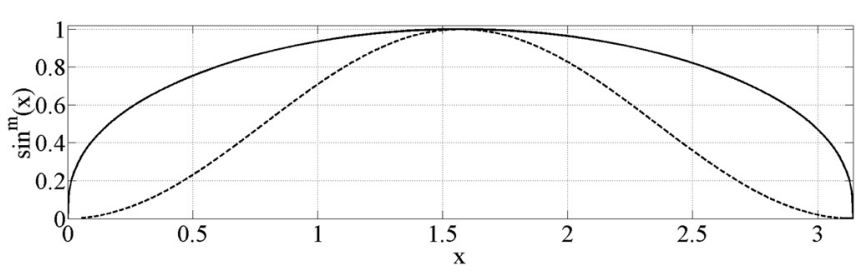

FIG. 4. Plot of $\sin ^{m}(x)$ for $m=2$ (dashed line) and for $m=0.385$ (solid line), based on the correlation in Eq. (4). The average values $\left\langle\sin ^{m}(x)\right\rangle$ $=(1 / \pi) \int_{0}^{\pi} \sin ^{m}(x) d x$ result in the well-known result of $1 / 2$ for $m=2$, as well as $0.802 \approx 4 / 5$ for $m=0.385$. 
modulation is that there is a time lag introduced by the wire's thermal inertia. The combination of the convective thermal resistance, $R_{t h}$, and the wire's heat capacity, $C_{\text {wire }}$, produce an exponential thermal equilibration time constant, $\tau_{t h}=R_{t h} C_{\text {wire }}$, for the temperature of the wire to respond to changes in either the rate of the convective cooling or the rate of heating. Using the wire's dimensions, the specific heat of nichrome, $c_{N i C r}=450 \mathrm{~J} / \mathrm{kg} / \mathrm{K}$, and the wire's mass density, $\rho_{N i C r}=8250 \mathrm{~kg} \mathrm{~m}^{-3}$, the thermal time constant is calculated in Eq. (7),

$$
\tau_{\text {th }}=R_{t h} C_{\text {wire }}=\rho_{\mathrm{NiCr}} c_{\mathrm{NiCr}} R_{t h} \frac{\pi D^{2} L}{4} \cong 0.9 \mathrm{~s} .
$$

It is worth noting that this thermal relaxation time, $\tau_{t h}$, is rather close to the observed modulation periods, like those shown in Fig. 3, and that the result for $h_{\text {eff }}$ in Eq. (6) is only meant to be representative, since it was evaluated for $v_{p}=2.0 \mathrm{~m} / \mathrm{s}$ (i.e., $\operatorname{Re} \cong 33$ ).

This lumped-element relaxation time analysis is valid if the temperature of the $\mathrm{NiCr}$ wire is not a function of the wire's radius. For this to be the case, the Biot number must be small: $\mathrm{Bi}<0.1$,

$$
\mathrm{Bi}=\frac{h_{\mathrm{eff}} L_{c}}{\kappa_{N i C r}}, \quad \text { where } L_{c} \equiv \frac{V}{A_{\text {surf }}} .
$$

$L_{c}$ is a characteristic length scale. For a cylinder, $L_{c}=D / 4$. For a nichrome wire, $\kappa_{N i C r}=11.3 \mathrm{~W} / \mathrm{m} / \mathrm{K}$, so $\mathrm{Bi} \cong 6 \times 10^{-7}$ $\ll 0.1$, thus ensuring that the wire's temperature is independent of the radial position and supporting the use of a lumped-element approach to the calculation of the thermal time constant that appears in Eq. (7). ${ }^{32}$

Using this analysis of the convective heat transfer process, it is possible to write an expression for the mean wire temperature, $T_{\text {wire }}$, in terms of the electrical power heating the wire, $\Pi_{e l}$, and the peak transverse velocity of the wire, $v_{p}$. Using Eqs. (4), (5), and (6), and assuming the same nominal thermophysical parameters for air at $20^{\circ} \mathrm{C}$, it is possible to write the effective convective heat transport coefficient,

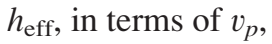

$$
\begin{aligned}
& \mathrm{Nu}=0.812 \operatorname{Re}^{0.385}=2.34 v_{p}^{0.385}\left(\frac{s}{m}\right)^{0.385} \\
& \Rightarrow h_{\mathrm{eff}}=0.082 \frac{W}{{ }^{\circ} \mathrm{C}}\left(\frac{s}{m}\right)^{0.385} v_{p}^{0.385} \equiv h_{0} v_{p}^{0.385} .
\end{aligned}
$$

At steady-state (i.e., for $t \gg \tau_{t h}$ ), the difference between the wire's average temperature, $T_{\text {wire }}$, and the air temperature far from the wire, $T_{\infty}$, can be related to the wire's peak velocity, $v_{p}$,

$$
\Delta T=T_{\text {wire }}-T_{\infty}=\frac{\Pi_{e l}}{h_{0} v_{p}^{0.385}} .
$$

Under operating conditions that exhibit amplitude modulation, a typical current through the wire might be $i_{a c}$ $=0.91 A_{a c}$. With $R_{d c} \cong 12 \Omega$, the total time-averaged power dissipation in the wire is $\Pi_{e l}=R_{d c} i_{a c}^{2} \cong 10 \mathrm{~W}$. Figure 5 is a

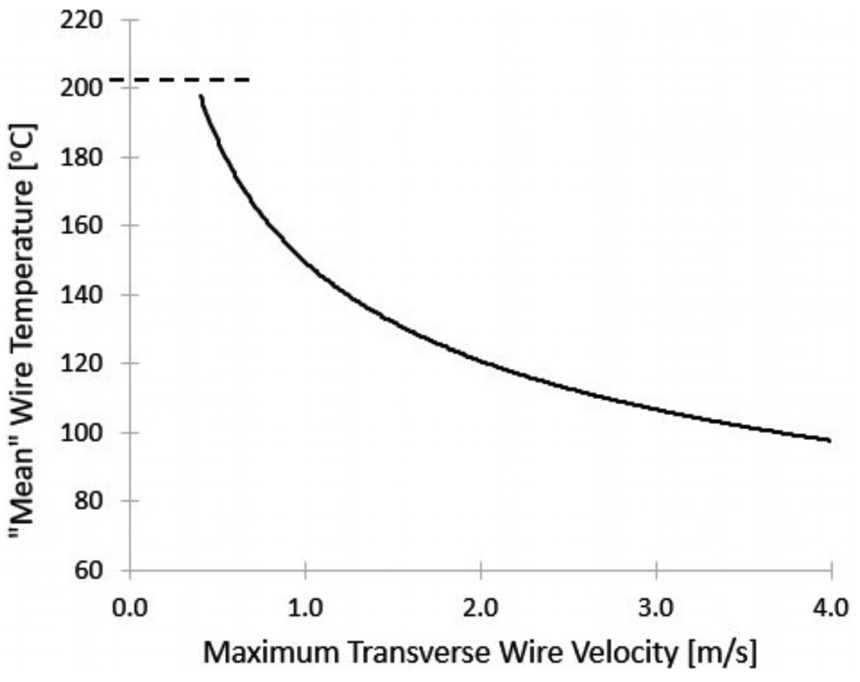

FIG. 5. Theoretical variation in the wire's average steady-state mean temperature, $T_{\text {wire }}$, as a function of its maximum transverse velocity, $v_{p}$, for an input electrical power of $\Pi_{e l}=10 \mathrm{~W}$ and the convective cooling as specified in Eq. (9). The dashed horizontal line intersects the vertical axis at the temperature determined by buoyancy-driven free convective cooling.

plot of the wire's average steady-state temperature, $T_{\text {wire }}$, as a function of the wire's maximum transverse velocity, $v_{p}$. The curve can be expressed as a power-law function of $v_{p}$,

$$
T_{\text {wire }}=146 v_{p}^{-0.321} \text {. }
$$

In Eqs. (10) and (11), the temperatures are expressed in degrees Celsius. The velocity's exponent is not -0.385 because $T_{\infty} \neq 0{ }^{\circ} \mathrm{C}$. The dashed horizontal line intersects the vertical axis at the temperature determined by buoyancydriven free convective cooling.

The behavior of the heated wire's temperature was confirmed in two ways: (1) Having carefully measured the wire's temperature coefficient of resistivity, $\left(1 / R_{0}\right)\left(d R_{N i C r} / d T\right)$ $=(1.576 \pm 0.001) \times 10^{-4} /{ }^{\circ} \mathrm{C}$ for $32^{\circ} \mathrm{C}<T_{\text {wire }}<143{ }^{\circ} \mathrm{C}$, measurement of the wire's electrical resistance is used to determine the wire's mean temperature. (2) The temperature distribution along the wire was determined using a highspeed infrared camera. ${ }^{25}$ Although the absolute temperature measured in this way is subject to the assignment of a wavelength-independent emissivity, the wire was sprayed with a heat-resistant black spray paint ${ }^{33}$ so that the colortemperature conversion could be more reliably based on an assumed emissivity of 1 .

Twenty-four infrared video recordings were made of the wire under various conditions. Two infrared camera screencapture images are shown in Fig. 6. The wire was at rest in the upper image and it was vibrating at its fundamental halfwavelength resonance frequency when the lower image was recorded.

For the quiescent wire, the coolest section was closest to the rigid attachment point since some of the heat was able to conduct to the boundary, making the temperature of that section of the wire be approximately $120^{\circ} \mathrm{C}$, based on the color palette below the upper image. Farther from the attachment point, the wire's temperature is hotter and uniform at the (optical) temperature of about $160^{\circ} \mathrm{C}$. According the 


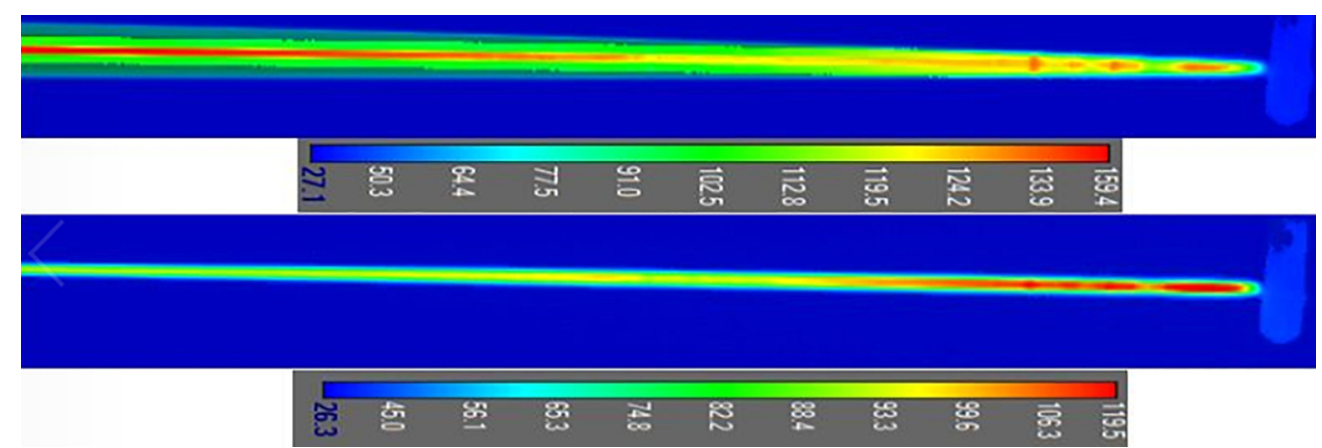

FIG. 6. (Color online) Two infrared images of a $16 \mathrm{~cm}$ long segment of the wire acquired with the high-speed infrared camera when the wire was driven by an $0.899 A_{a c}$ alternating current at $171.0 \mathrm{~Hz}\left(\Pi_{e l}=10.5 \mathrm{~W}\right)$. The rigid attachment rod is visible at the right in both images. (Upper) This is the wire's temperature distribution before the wire starts vibrating. Note that the hottest (red) section of the wire, which is at approximately $160{ }^{\circ} \mathrm{C}$ (optical), is farthest from the attachment point. (Lower) When the wire is vibrating, convective cooling makes the portion of the wire farthest from the attachment cooler, at approximately $70^{\circ} \mathrm{C}$ (optical), while the nearly stationary portion of the wire near the attachment point is the hottest portion, reaching approximately $120^{\circ} \mathrm{C}$ (optical).

manufacturer's specifications the quiescent wire's temperature, when passing a current of $0.9 A_{a c}$, should have been about $205^{\circ} \mathrm{C}$ (see Fig. 5). At that current, the electrical resistance measurement inferred a spatially averaged wire temperature of $200^{\circ} \mathrm{C}$ that was based on a best-fit to the electrical resistance vs current of $R(i)=0.409(\Omega / A) i+13.0 \Omega$ with a relative uncertainty in the slope of $\pm 5.2 \%$, for $0.2 A_{a c}$ $<i<1.0 A_{a c}$. Given the variation of temperature visible in Fig. 6 (upper), it is conceivable that the manufacturer's temperature estimate for the quiescent wire and the spatially averaged temperature inferred from the electrical resistance measurement are in quite good agreement and that an assumed emissivity of 1 used to infer the "optical" temperature is too high.

As seen in Fig. 6 (lower), the temperature distribution along the vibrating wire is changed substantially from its quiescent state. When the wire is vibrating in its fundamental half-wavelength mode, the hottest portion of the wire is the part closest to the boundary at roughly $120^{\circ} \mathrm{C}$ and the (optical) temperature farther from the boundary is only $70^{\circ} \mathrm{C}$. This is consistent with the fact that the velocity nodes glow and the other portions of the wire disappear when this apparatus is used as a lecture demonstration. ${ }^{2}$ Based on the typical convective cooling curves, like the example shown in Fig. 5, which was calculated based on an electrical power dissipation of $10 \mathrm{~W}$, it also appears that the use of an assumed emissivity of 1 is too high, rendering the indicated absolute optical temperature measurement values too low.

\section{EXPERIMENTALLY DETERMINED RESONANCE FREQUENCY SHIFTS AND ADIABATIC INVARIANCE}

As with the previous studies of large-amplitude standing waves on strings mentioned in the Introduction, ${ }^{6,7}$ the heated wire's fundamental resonance frequency is amplitude dependent. Like those earlier investigations, the heated wire's resonance frequency increases with increasing amplitude, although the primary contribution to the increase in resonance frequency is due to thermal effects (i.e., convective cooling) rather than just the nonlinear time-averaged increase in the arc length of the string between the rigidly fixed boundaries.
The most significant change in the resonance frequency is due to thermal effects. For the heated wire, an increase in the wire's mean temperature leads to a decrease in the wire's fundamental resonance frequency. Two such sets of measurement of that resonance frequency reduction as a function of increasing current are shown in Fig. 7.

This new effect is a consequence of the fact that the pulley imposes an interesting boundary condition and the wire's length is also coupled to the height of the tensioning mass through the wire's linear coefficient of thermal expansion, $\alpha_{N i C r} \cong 1.7 \times 10^{-5} /{ }^{\circ} \mathrm{C}$, therefore the wire also has to be considered as a primitive (i.e., very inefficient) heat engine: The pulley acts as a rigidly fixed boundary for the wire's transverse vibratory motion, which occurs at the driving frequency, $f$, but it is also a low-pass filter for equilibration of the mean tension in the wire due to the force that the mass provides to maintain that mean tension. The pulley

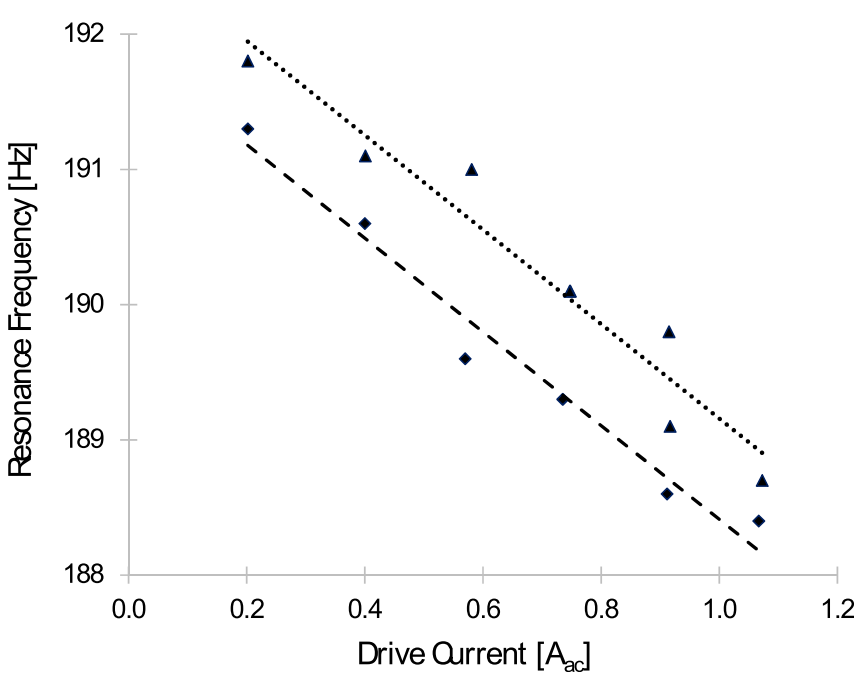

FIG. 7. (Color online) Two experiments that measured resonance frequency as a function of the drive current both demonstrate that the resonance frequency decreases with increasing drive current. Both experiments show that the rate of frequency decrease, $d f / d i \cong-3.5 \mathrm{~Hz} / A_{a c}( \pm 10 \%)$. In these measurements, the tensioning mass was $1.5 \mathrm{~kg}$. This result is contrary to the expectation of increasing frequency with increasing current if the current caused a decrease in $\rho_{L}$ due to a positive linear thermal expansion coefficient, $\alpha_{N i C r}$, and a positive Poisson's ratio, $\sigma$, as shown in Eq. (23). 
guarantees that the time-averaged tension in the wire, $\mathcal{T}$, remains constant, $\mathcal{T}=M g$.

The wire's resonance frequency is therefore determined by both its vibrational amplitude and the work done on the wire by the mass as it lowers or the work done on the mass by the wire as it raises. These dynamical and thermal influences on the wire's resonance frequency can be understood by invoking the Boltzmann-Ehrenfest adiabatic principle. ${ }^{34}$

\section{A. Resonance frequency shifts}

The amplitude of the wire's vibration depends upon the difference between the drive frequency, $f$, and the wire's natural frequency, $f_{1}$. As just mentioned, and as illustrated in Fig. 7, the natural frequency is a function of the drive current and therefore the temperature of the wire. Since $f_{1}$ decreases as the wire is heated and the drive frequency is less than $f_{1}$, the amplitude of the wire's vibration increases as the separation of $f$ and $f_{1}$ becomes smaller.

In all of the experiments reported here, the wire was driven by a constant alternating current, $i(t)$, at a constant frequency, $f$. If the fundamental half-wavelength resonance frequency, $f_{1}$ (also known as the "natural frequency") changes, then the amplitude of oscillation will change according to the "Rayleigh line shape" that relates the amplitude, $A\left(f / f_{1}\right)$, to the frequency ratio, $f / f_{1}$, and to the quality factor of the fundamental resonance, $Q_{1}$,

$$
A\left(f / f_{1}\right)=\frac{A_{\max } / Q_{1}}{\left[\left(\frac{f}{f_{1}}-\frac{f_{1}}{f}\right)^{2}+\frac{1}{Q_{1}^{2}}\right]^{1 / 2}} .
$$

Such amplitude variations due to a change in $f_{1}$ while $f$ is held fixed are shown schematically in Fig. 8. To simplify analysis of the amplitude modulation shown in Fig. 8, the

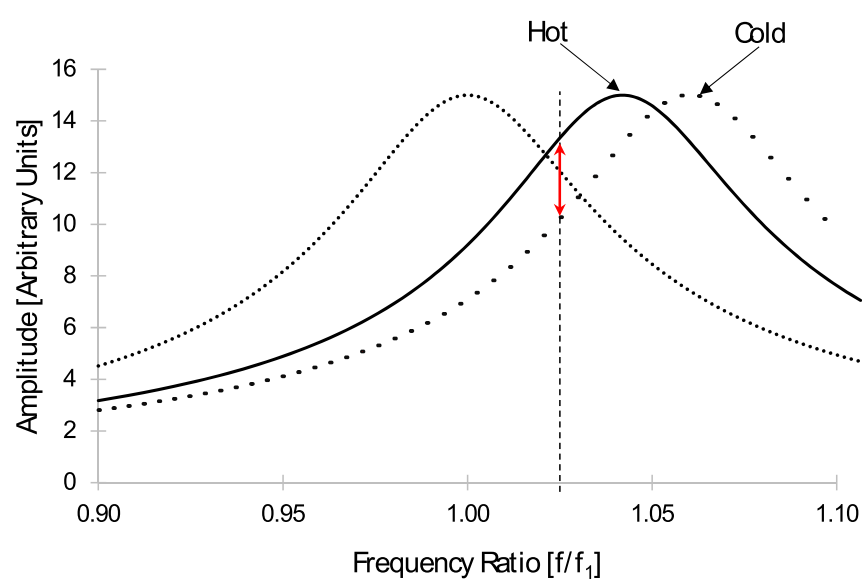

FIG. 8. (Color online) Three (linear) resonance response curves shown here are intended to describe the response of the vibrating wire driven at a constant frequency, $f$, when the resonance frequency, $f_{1}$, changes due to the wire's temperature. To make the figures easier to visualize, all the curves have $Q_{1}=15$. The close dotted curve is the low-amplitude linear response result with its peak at $f / f_{1}=1.00$, with $f_{1}=c_{t} / 2 L$. The solid curve could represent the wire in the absence of convective cooling (hot). If the wire is cooled, the wide-spaced dotted curve (cold) would represent the wire's response. The red arrow shows the amplitude modulation produced if the wire is driven at fixed frequency, $f / f_{1} \cong 1.025$. rate-of-change of amplitude with frequency, $d A / d f$, can be approximated by fitting the Rayleigh line shape to a triangular function, as shown in Fig. 9.

The sensitivity of the amplitude, $A$, to changes in the drive frequency, $f$, close to the peak resonance response, can be related to the quality factor of the fundamental resonance, $Q_{1}$, and to the drive frequency ratio, $f / f_{1}$. As shown in Fig. 9, the slope of a resonance response curve for normalized amplitudes, $A\left(f / f_{1}\right) / A(0)$, where $A(0)$ is the zero-frequency response to the forcing, are represented to an adequate approximation by two straight lines for $A\left(f / f_{1}\right) / A(0) \geq Q / 4$,

$$
\begin{aligned}
& \frac{d(A / A(0))}{d\left(f / f_{1}\right)} \cong \frac{Q^{2}}{2}=\frac{f_{1}}{A(0)} \frac{d A}{d f} \\
& \Rightarrow \frac{d A}{d f} \cong \frac{A(0)}{2 f_{1}} Q^{2}=\frac{2 A\left(f_{1}\right)}{f_{1}} Q, \text { if } \frac{A(f)}{A(0)} \geq \frac{Q}{4} .
\end{aligned}
$$

If $Q_{1}=500$ and $f_{1}=\left(10^{3} \mathrm{rad} / \mathrm{s}\right) / 2 \pi \cong 160 \mathrm{~Hz}$, and if $A\left(f_{1}\right)=z_{p}=3 \mathrm{~mm}$, then $d A / d f=d z_{p} / d f \cong 4.7 \mathrm{~mm} / \mathrm{Hz}$.

\section{B. Resonance quality factor and amplitude relaxation time}

The quality factor of the fundamental standing wave resonance of the wire was measured under two different conditions. The first measurement used the first apparatus. The wire was driven at resonance and then was disconnected (electrically) from the drive amplifier and allowed to decay freely. The wire's transverse velocity amplitude was measured using the laser-Doppler velocimeter ${ }^{22}$ and recorded using a digital oscilloscope.

The digitized time-record of the velocimeter's output during that decay measurement consisted of 10990 pairs of points (time and velocity) spanning $2.75 \mathrm{~s}$. The record was imported to an Excel spreadsheet that used a multi-variable "solver" program to minimize the square difference between the measured points and a simple exponentially decaying oscillatory voltage characterized by five parameters: $B, A, \omega$, $\phi$, and $\tau$,

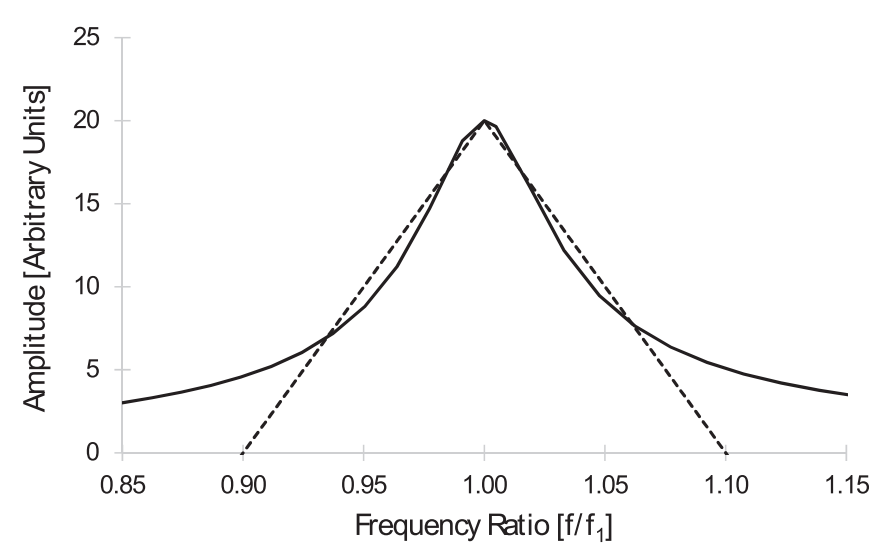

FIG. 9. To simplify calculations of the wire's response to a change in the frequency ratio, $f / f_{1}$, the resonance response curve of Eq. (12) (solid) can be represented by the two straight dashed lines. As shown in this example, with $Q_{1}=25$, the amplitude given by the linear approximation is quite close to the resonance response for relative amplitudes, $A\left(f / f_{1}\right) / A(0)$, larger than $Q_{1} / 4$. 


$$
V(t)=B+A \sin (\omega t+\phi) e^{-t / \tau}
$$

The best fit to Eq. (14) produced $B=0.00 \mathrm{~V}, A=0.213 \mathrm{~V}$, $\omega=994.4 \mathrm{rad} / \mathrm{s}, \phi=0.783 \mathrm{rad}$, and $\tau=2.46 \mathrm{~s}$. Based on the ratio of the minimized square error, $\Sigma(\text { error })^{2}=1.02 \mathrm{~V},{ }^{2}$ and the square sum of all of the data points, $\Sigma(\text { data })^{2}=208.4 \mathrm{~V}^{2}$ the relative uncertainty of the fit parameters is about $\pm 1 / 4 \%$. The most significant result of that least-squares fit is the determination of the exponential relaxation time, $\tau_{\text {free }}=2.46 \mathrm{~s}$. That relaxation time is related to the free-decay quality factor of the fundamental mode of vibration, $Q_{1}$,

$$
Q_{1}=\pi f_{1} \tau
$$

Thus, the free-decay quality factor is $Q_{1}($ free $)=1223$.

The free-decay quality factor represents the wire's maximum quality factor because the decay took place while the wire was in an open-circuit condition. To determine the quality factor for the driven system, it is necessary to drive the wire so that there is a closed-circuit path that allows the electrodynamic dissipation factors to contribute to the characteristic exponential decay time, $\tau$. For that measurement, the second apparatus was used and the wire's displacement amplitude as a function of the drive frequency was measured using the thin-beam load cell shown in Fig. 1(b).

As shown in Fig. 10, this lower value of $Q_{1}=106$ $\ll Q_{1}$ (free) corresponds to an exponential relaxation time $\tau \cong 0.2 \mathrm{~s}$ based on Eq. (15). This is shorter than the calculated example for the wire's thermal equilibration time, $\tau_{t h} \cong 0.9 \mathrm{~s}$, based on Eq. (7). If $\tau_{t h} \gg \tau$ for all experimental conditions, then it is the thermal equilibration time that will dominate the dynamics of the amplitude modulation.

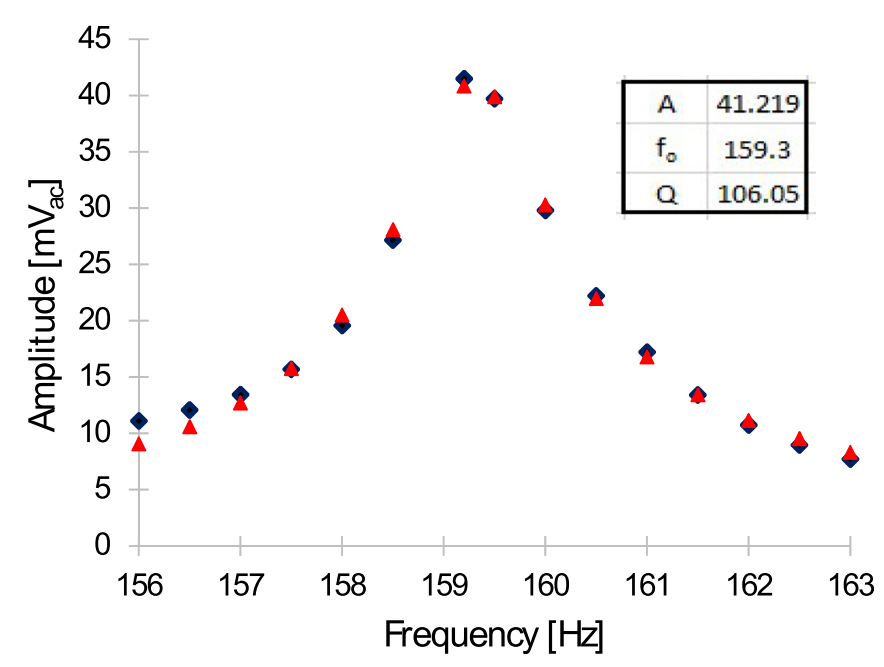

FIG. 10. (Color online) Low-amplitude response curve for the electrodynamically driven wire. The black diamonds are the wire's response as measured by the thin-beam load cell shown in Fig. 1(b). The red triangles are the values of the Rayleigh line shape of Eq. (12) at the same frequencies that has been fit to the data using a nonlinear curve-fitting algorithm that minimizes the sum of the squares of the differences between the data and the Rayleigh line shape. The resonance parameters that are the results of the fitting process are shown in the inset. Based on the ratio of the minimized square error, $\Sigma(\text { error })^{2}=10.5 \mathrm{mV}$ (Ref. 2 ), and the square sum of the data points, $\Sigma(\text { data })^{2}=289 \mathrm{mV}$ (Ref. 2 ), the relative uncertainty of the fit parameters is about $\pm 1.7 \%$.

\section{Amplitude dependent frequency shifts}

To apply adiabatic invariance to the change in the vibrating wire's natural frequency, expressions must be developed to relate changes in wire's length due to vibrational amplitude and to the wire's thermally induced length changes due to changes in the wire's mean temperature. The vibration of a fixed-fixed wire is known to exhibit nonlinear "stiffening" that is due to the increase in the average tension produced by the increase in the wire's length. ${ }^{35}$ This arc length increase can be calculated for a normal mode (i.e., at a frequency where the quiescent length of the wire, $L$, corresponds to an integer number of half-wavelengths) from the peak amplitude of transverse vibration, $z_{p}$. The increase in the length of the wire, $\delta L$, can be calculated in terms of the wire's "speaking length," $L$ (i.e., the distance between the rigid end supports) and integration of the square of the wire's slope at all positions along the wire,

$$
\delta L=\frac{1}{2} \int_{0}^{L}\left(\frac{\partial z}{\partial x}\right)^{2} d x=\frac{\pi^{2}}{4} \frac{z_{p}^{2}}{L} \cong 2.47 \frac{z_{p}^{2}}{L} .
$$

Since $z(x=L / 2, t)$ is a sinusoidally varying function of time, i.e., $z(L / 2, t)=z_{p} e^{j \omega t}$, the time-averaged length change, $\overline{\delta L}$, is half of the result provided in Eq. (16),

$$
\overline{\delta L}=\frac{\pi^{2}}{8} \frac{z_{p}^{2}}{L} \cong 1.23 \frac{z_{p}^{2}}{L} .
$$

The time-averaged tension of the wire in our apparatus remains constant, independent of the vibrational amplitude, $z_{p}$, because that tension is provided by a mass through the pulley. Yet, a tuning curve for the heated wire, such as the example provided in Fig. 2, clearly exhibits "nonlinear stiffening" and the "tuning hysteresis" that is also characteristic of a fixed-fixed wire. For an assumed peak amplitude of $z_{p}=2.0 \mathrm{~mm}$, the time-averaged amplitude-induced length change is $\overline{\delta L} \cong 9.4 \mu \mathrm{m}$.

Since the overall length of the wire, from the fixed end to the attachment point of the mass, does not change, this vibration-induced arc length increase, $\overline{\delta L}$, must be accompanied by a rise in the height of the tensioning mass. The vibration is therefore doing an amount of work, $W$, on the mass, $W=M g \overline{\delta L} \cong 9.2 \times 10^{-5} \mathrm{~J}$. Adiabatic invariance suggests that the wire's half-wavelength resonance frequency will increase (see the Appendix).

The maximum kinetic energy of the wire's vibration for $z_{p}=2.0 \mathrm{~mm}$ depends upon the mass, $m_{s}$, of the vibrating portion of the wire,

$$
(K E)_{\max }=\frac{m_{s} v_{p}^{2}}{4}=\frac{\rho_{L} L\left(\omega z_{p}\right)^{2}}{4} \cong 3.8 \times 10^{-4} \mathrm{~J} .
$$

If this were the only energy that was needed to define the energy $E$ in the denominator of Eq. (A1) then $\delta f / f_{1}=22 \%$, which is clearly too large. Because it is not yet clear what energies must be included in the value of $E$, the relative magnitude of this frequency increase, $\delta f / f_{1}$, cannot be calculated, although its sign must be positive. Further 
experiments should be conducted to determine the correct value of $E$ for a system that involves both mechanical and thermal energies.

This resonance frequency increase predicted by adiabatic invariance is opposite in sign to the frequency decrease predicted by Gottlieb who claims that for a constant tension string at a fixed temperature, the resonance frequency decreases with increasing transverse vibrational amplitude. ${ }^{35}$ Gottlieb's mathematical analysis of the constant tension string produces an expression for the resonance frequency shift, $\left(\delta f / f_{1}\right)_{\text {Gott }}$ that depends upon the amplitude of vibration, $z_{p}$,

$$
\left(\frac{\delta f}{f_{1}}\right)_{\text {Gott }} \cong-\frac{3}{16}\left(\frac{\pi z_{p}}{L}\right)^{2}
$$

The approximate expression to his full result in Eq. (19) relies on the fact that $z_{p} / L \ll 1$, so Gottlieb's higher-order correction, proportional to $\left(\pi z_{p} / L\right),{ }^{4}$ can be ignored and a binomial expansion could be used to extract the $\left(\delta f / f_{1}\right)_{\text {Gott }}$ from his more complete expression.

Gottlieb suggests one interpretation of this decrease in resonance frequency for an unheated wire, which is that the wire's vibration-induced curvature increases the "effective length" of the wire by the amount, $\overline{\delta L}$, calculated in Eq. (17): "This is not unexpected, since the vibrating length (with constant density) is increased by the curvature."

That average length increase, in conjunction with Eq. (1), would lead to a decrease in resonance frequency, $f_{1}$, with increasing vibrational amplitude for the constant-tension wire. In our experiment, this length change, corresponding to $z_{p}=2.0 \mathrm{~mm}$, results in a relative decrease in the resonance frequency using Gottlieb's effective length interpretation,

$$
\frac{\delta f}{f_{1}}=\frac{-\overline{\delta L}}{L} \quad \text { for } z_{p}=2.0 \mathrm{~mm}, \frac{\delta f}{f_{1}}=-1.8 \times 10^{-5} .
$$

For the same conditions used to evaluate the relative frequency decrease in Eq. (20), Gottlieb's expression in Eq. (19) produces a somewhat larger relative frequency decrease: $\left(\delta f / f_{1}\right)_{\text {Gott }}=-2.7 \times 10^{-5}$. In addition to this discrepancy, Gottlieb provides no experimental confirmation for the results of his calculation showing that the resonance frequency decreases as a function of increasing vibrational amplitude.

Since our concern is the periodic modulation of the wire's amplitude, we are more interested in the shift of the wire's fundamental resonance frequency as the amplitude varies. In going from $z_{p}=2.0 \mathrm{~mm}$ to $z_{p}=3.0 \mathrm{~mm}$, the arc length increases by $1.2 \times 10^{-5} \mathrm{~m}$, based on Eq. (16), so the tensioning mass is raised by that amount and adiabatic invariance requires the resonance frequency to increase. For a reduction in amplitude from $z_{p}=2.0 \mathrm{~mm}$ to $z_{p}=1.0 \mathrm{~mm}$, the time-averaged length change is $+7.0 \times 10^{-6} \mathrm{~m}$, resulting in a decrease in the tensioning mass's height and a corresponding decrease in the resonance frequency.

As we are about to demonstrate, the amplitudedependent change in the height of the tensioning mass is insignificant in comparison to the thermally induced motion of the tensioning mass.

\section{Length changes with temperature}

Thus far, neither Eq. (17) nor Eq. (19) addresses the thermal effects that are present for our heated wire. To evaluate the change in the wire's length due to temperature changes, it is necessary to examine both the time-averaged length change due to the wire's temperature change with amplitude, as illustrated in Fig. 5, and the effect that such a temperature induced length change, $\overline{\delta L}_{t h}$, has on the linear mass density of the wire, $\rho_{L}$. Both of those length-related changes are mediated by the wire's linear coefficient of thermal expansion, $\alpha_{\mathrm{NiCr}}=1.7 \times 10^{-5} /{ }^{\circ} \mathrm{C}$.

The thermally induced value of $(\delta L / L)$ can be expressed in terms of the amplitude of the wire's oscillations from an expression like Eq. (11) that is based on the convective cooling model of Eqs. (9) and (10) which produces a timeaveraged temperature change, $\delta T_{\text {wire }}$, resulting in $\overline{\delta L}_{t h} / L$ $=\alpha_{N i C r} \times \delta T_{\text {wire }}$.

Again, evaluating the thermally induced frequency shifts for changes in the vibrational amplitude that are typical for the observed amplitude modulations, in going from $v_{p}=\omega z_{p}$ $=2.0 \mathrm{~m} / \mathrm{s}$ to $v_{p}=\omega z_{p}=3.0 \mathrm{~m} / \mathrm{s}$, using the expression for averaged wire temperature, $T_{\text {wire }}$, in Eq. (11), the length change produced by this temperature change, $\delta T_{\text {wire }}=-14.3^{\circ} \mathrm{C}$, makes $\left(\overline{\delta L}_{t h} / L\right)=-2.4 \times 10^{-4}$. Such a change would raise the tensioning mass by nearly $1.3 \times 10^{-4} \mathrm{~m}$, thereby doing $1.25 \times 10^{-3} \mathrm{~J}$ of work raising a $1 \mathrm{~kg}$ mass and therefore raising the resonance frequency. That amount of work is nearly 14 times greater than the work done to raise the tensioning mass due to the time-averaged arc length increase calculated under the same circumstances but based on Eq. (17).

For a reduction in velocity amplitude from $v_{p}=\omega z_{p}$ $=2.0 \mathrm{~m} / \mathrm{s}$ to $v_{p}=\omega z_{p}=1.0 \mathrm{~m} / \mathrm{s}$, the magnitude of the thermal effect is even larger: $\delta T_{\text {wire }}=+29.1^{\circ} \mathrm{C}$ so $\left(\overline{\delta L}_{t h} / L\right)=+5.0$ $\times 10^{-4}$. Such a change would lower the tensioning mass by nearly $2.6 \times 10^{-4} \mathrm{~m}$, extracting $2.55 \times 10^{-3} \mathrm{~J}$ of work by lowering a $1 \mathrm{~kg}$ mass and therefore lowering the resonance frequency. The magnitude of that length change does almost 30 times more work on the tensioning mass than was done by the time-averaged arc length increase, calculated under the same circumstances, but based on Eq. (17). For that reason, the isothermal Gottlieb discrepancy is not significant for our understanding of the amplitude modulation process.

The thermally induced length change also impacts the resonance frequency shift because an increase in length will also change the wire's linear mass density, $\rho_{L}$. In accordance with Eq. (1), that linear mass density change will influence the resonance frequency with $L$ and $\mathcal{T}$ both held constant,

$$
\ln \left[f_{1}\right]=-\frac{1}{2} \ln \left[\rho_{L}\right]+\ln [\text { constant }] \Rightarrow\left(\frac{\delta f}{f_{1}}\right)_{\sigma}=-\frac{1}{2} \frac{\delta \rho_{L}}{\rho_{L}}
$$

Poisson's ratio, $\sigma$, relates the change in the wire's diameter to its change in length,

$$
\begin{array}{r}
\rho_{L}=\rho_{N i C r} \frac{\pi D^{2}}{4}, \text { and } \frac{\delta D}{D}=-\sigma \frac{\overline{\delta L}_{t h}}{L}, \\
\text { so } \frac{\delta \rho_{L}}{\rho_{L}}=-2 \sigma \frac{\overline{\delta L}_{t h}}{L} .
\end{array}
$$


Combining the results of Eqs. (21) and (22), the additional relative change in resonance frequency, $\left(\delta f / f_{1}\right)_{\sigma}$, will be opposite in sign to the changes in length that cause the tensioning mass's position to change,

$$
\left(\frac{\delta f}{f_{1}}\right)_{\sigma}=-\frac{1}{2} \frac{\delta \rho_{L}}{\rho_{L}}=+\sigma \frac{\overline{\delta L}_{t h}}{L}=\sigma \alpha_{N i C r} \delta T_{\text {wire }}
$$

According to the manufacturer's specification, ${ }^{18}$ and other sources, ${ }^{36} \sigma_{\mathrm{NiCr}} \cong 0.3$ so the direct thermal expansion dominates the overall thermally induced linear mass density change, but without a reliable value for $E$ to use in the denominator of the expression for adiabatic invariance in Eq. (A1), it is not yet possible to make a direct comparison of the linear mass density and thermally induced overall length change effect.

\section{MEASUREMENTS OF AMPLITUDE MODULATION}

Having identified the relevant time constants and developed expressions that relate the change in resonance frequency to changes in wire temperature, it is possible to enhance the introductory qualitative argument provided in Sec. I C regarding the observed amplitude modulation in the electrodynamically driven "burning wire" demonstration.

The modulation experiments reported in this paper always follow a sequence that starts by setting the drive current's amplitude and slowly increasing the drive frequency while observing the increase in the wire's transverse displacement as the wire's resonance frequency is approached from below. The amplitude increases follow the frequency increases quickly since the time constant for the wire to reach its steady-state resonant amplitude, $\tau$, is fairly short, being on the order of a few hundred milliseconds or less based on $Q_{1}$ and $\omega$, as expressed in Eq. (15).

Because the thermal equilibration time constant, $\tau_{t h}$, as expressed in Eq. (7), is several times longer than $\tau$, when the wire's vibrational amplitude reaches its maximum value it takes additional time for the increased convective cooling to lower the wire's mean temperature, $T_{\text {wire }}$, due to the wire's thermal inertia. As the wire's mean temperature decreases, the tensioning mass rises (due to thermal contraction) and the resonance frequency increases. In effect, $\tau_{t h}$ becomes a retardation time; the amplitude of the wire's vibration at time $t$ corresponds to the value of $f / f_{1}$ at the earlier time, $t-\tau_{t h}$.

The rise in resonance frequency decreases the frequency ratio, $f / f_{1}$, and therefore reduces the wire's vibrational amplitude which, in turn, reduces the convective cooling causing the wire's mean temperature to increase, the mass to descend, and the resonance frequency to be reduced, again on a time scale determined by $\tau_{t h}$.

Once the wire becomes hotter, $f / f_{1}$ is again brought closer to one and the amplitude starts to increase, just as it did when this qualitative description started. We observed those amplitude modulations to repeat regularly and indefinitely.

Because $\tau<\tau_{t h}$, it is the thermal equilibration time constant that determines the modulation rate. The amplitude of the modulation is determined by the combination of the resonance quality factor, as approximated in Eq. (13), and by the magnitude of the resonance frequency shift with the wire's mean temperature.

By changing the force that pushes the two ball bearing races together in the third apparatus, visible at the bottom of Fig. 1(c), and surrounding the apparatus with a cardboard tube to suppress the random air motion in the lab from influencing the wire, it was possible to produce $100 \%$ modulation as shown by the time record of the LVDT's plunger position shown in Fig. 11.

In Fig. 11, the wire was tensioned with a mass of $0.75 \mathrm{~kg}$. It was driven by a current of $0.798 A_{a c}$ at $135.2 \mathrm{~Hz}$. The change in the position of the mass was dominated by the large change in the wire's temperature between the time when it was completely at rest, so the wire was hottest and at its maximum elongation, to the time when it was vibrating so that convective cooling reduced the average wire temperature thus raising the tensioning mass.

The value of the maximum change in the wire's length, for the measurement shown in Fig. 11, is $540 \mu \mathrm{m}$. With $\alpha_{\mathrm{NiCr}}$ $=17 \mathrm{ppm} /{ }^{\circ} \mathrm{C}$ and $L=0.525 \mathrm{~m}$, that length change corresponds

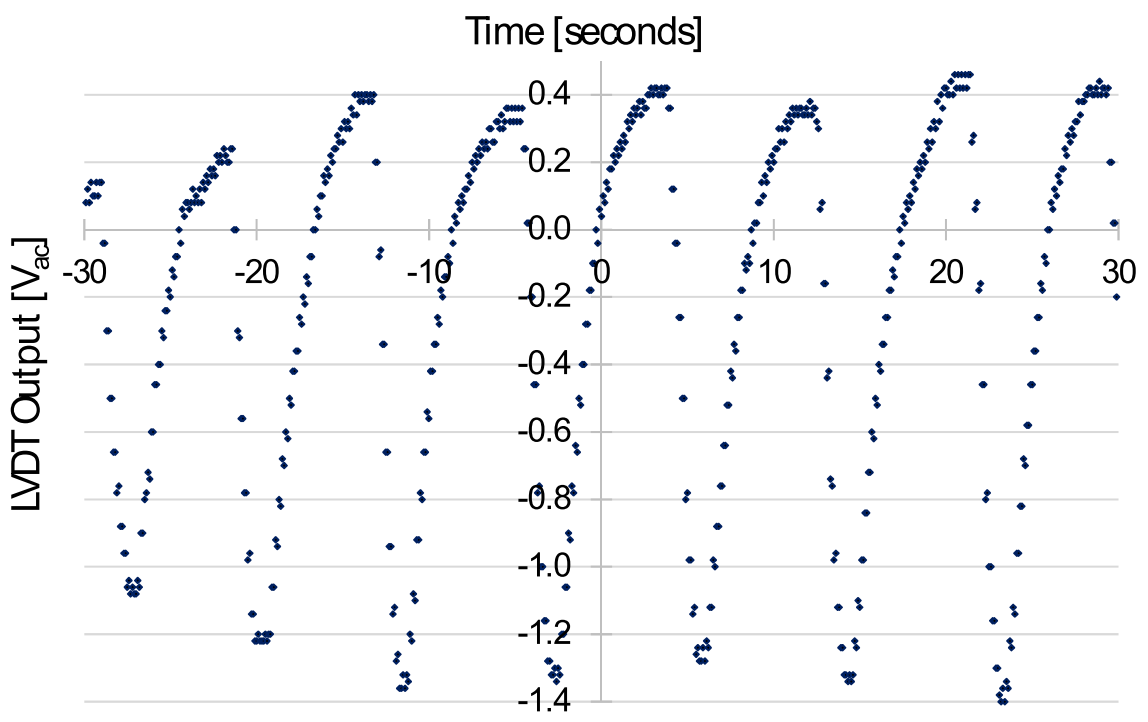

FIG. 11. (Color online) Using a lockin amplifier to rectify the ac signal produced by the LVDT's secondary winding, the motion of the tensioning mass was observable throughout the $8.5 \mathrm{~s}$ modulation period shown. The lockin's output was applied to a digital storage oscilloscope to produce this time record of the mass's motion. Correcting for the lock-in gain $(\times 20=+26 \mathrm{~dB})$, the span shown from -1.4 to $+0.4 \mathrm{~V}_{a c}$ corresponds to $\mathrm{a}$ change in the mass's height of $\Delta h=0.54 \mathrm{~mm}$, based on the calibration of the LVDT. 
to $\Delta T \cong 60^{\circ} \mathrm{C}$. The work the wire did upon cooling to produce that motion is $(\Delta E)_{\text {thermal }}=\operatorname{Mg}(\Delta h)=3.9 \times 10^{-3} \mathrm{~J}$. Again, if $z_{p}=3.0 \mathrm{~mm}$, then the time-averaged arc length change induced by the vibration is only $\overline{\delta L} \cong 20 \mu \mathrm{m}$ and the corresponding change in gravitational potential energy is only $(\Delta E)_{v i b r}=M g \overline{\delta L}=1.5 \times 10^{-4} \mathrm{~J}$.

\section{COUPLED DIFFERENTIAL EQUATIONS MODEL}

It is possible to produce a quantitative description of the heated wire's behavior while it is vibrating in its fundamental half-wavelength mode that accounts for the vibrationally enhanced convective heat exchange with the surrounding air and the impact that the wire's resulting temperature variations have on its resonance frequency. Such an analysis will begin with a lumped-element representation of the wire's vibration followed by the incorporation of the convective cooling, represented in Eqs. (4) and (9), as illustrated in Fig. 5, along with the resultant thermal time constant, $\tau_{t h}$, calculated in Eq. (7), incorporating the wire's heat capacity, $C_{\text {wire }}$.

These effects produce a set of coupled thermokinetic nonlinear differential equations that incorporate coefficients obtained from the experiments whose results were described previously. Because the time that characterizes the vibration, $(\omega)^{-1} \cong 1.0 \times 10^{-3} \mathrm{~s}$, is so much shorter than both the modulation period, $0.5 \mathrm{~s}<T_{\text {mod }}<10 \mathrm{~s}$, and the thermal equilibration time, $\tau_{\text {th }} \cong 1.0 \mathrm{~s}$, it is possible to use the multiple scale method (MSM), ${ }^{37}$ also known as the method of multiple time scales, to isolate the slow modulation of the wire's amplitude and then solve those equations numerically using a Runge-Kutta scheme.

Since those experimental parameters and thermophysical constants have been spread throughout, Table III is provided to summarize their values that will now be employed to demonstrate that all of the experimentally observed behaviors are exhibited by this mathematical model of an electrodynamically driven heated wire operating at its fundamental half-wavelength resonance.

\section{A. Lumped element oscillator}

In the linear approximation, the linearly polarized transverse displacement of the wire, $z(x, t)$, is described by the wave equation

$$
\partial_{t t} z+\eta \partial_{t} z-c_{t}^{2} \partial_{x x}^{2} z=0
$$

The factor, $\eta=\omega_{\infty} / Q$, has been introduced to account for all of the dissipative effects. Following Gough, ${ }^{38}$ Eq. (24) can be reduced to a simple harmonic oscillator equation if only solutions corresponding to the fundamental half-wavelength mode of vibration are examined by substituting $z(x, t)$ $=Z(t) \sin (\pi x / L)$,

$$
m_{s} \ddot{Z}+\eta m_{s} \dot{Z}+\left(\frac{\pi c_{t}}{L}\right)^{2} m_{s} Z=0 .
$$

Based on Eq. (1), the linear radian frequency at room temperature $T_{\infty}, \omega_{\infty}$, that is a solution to Eq. (25) in the absence of dissipation (with $\eta=0$ ) can be written as $\omega_{\infty}$ $=(\pi / L) \sqrt{M g / \rho_{L}}$, where, as before, $M$ is the mass which provides the wire's tension and $g$ is the gravitational acceleration. The mass of the vibrating portion of the wire is $m_{s}=\rho_{L} L$ and the effective stiffness is $k=m_{s}\left(\pi c_{t} / L\right)^{2}$, so $\omega_{\infty}=\sqrt{k / m_{s}}$. The damping coefficient can be related to the ratio of the linear

\begin{tabular}{|c|c|c|c|c|}
\hline Material & Parameter & Symbol & Value & Unit \\
\hline \multirow[t]{2}{*}{ Wire } & Diameter & $\mathrm{D}$ & 0.25 & $\mathrm{~mm}$ \\
\hline & Vibrational length & $\mathrm{L}$ & 52.5 & $\mathrm{~cm}$ \\
\hline \multirow[t]{12}{*}{$\mathrm{NiCr}$} & Composition: $\mathrm{Ni} 60 \%, \mathrm{Cr} 16 \%, \mathrm{Fe} 24 \%$ & & & \\
\hline & Mass density & $\rho_{\mathrm{NiCr}}$ & $8.25 \times 10^{3}$ & $\mathrm{~kg} \mathrm{~m}^{-3}$ \\
\hline & Linear mass density $=\rho_{\mathrm{NiCr}} \pi D^{2} / 4$ & $\rho_{L}$ & $4.05 \times 10^{-4}$ & $\mathrm{~kg} \mathrm{~m}^{-1}$ \\
\hline & Wire mass $=\rho_{L} L$ & $m_{s}$ & $2.13 \times 10^{-4}$ & $\mathrm{~kg}$ \\
\hline & Linear expansion coefficient & $\alpha_{\mathrm{NiCr}}$ & $1.7 \times 10^{-5}$ & $\mathrm{~K}^{-1}$ \\
\hline & Thermal conductivity & $\kappa_{\mathrm{NiCr}}$ & 11.3 & $\mathrm{~W} \mathrm{m^{-1 }} \mathrm{K}^{-1}$ \\
\hline & Specific heat & $c_{\mathrm{NiCr}}$ & 450 & $\mathrm{~J} \mathrm{~kg}^{-1} \mathrm{~K}^{-1}$ \\
\hline & Heat capacity $=\rho_{L} c_{N i C r} L$ & $C_{\text {wire }}$ & $9.6 \times 10^{-2}$ & $\mathrm{~J} \mathrm{~K}^{-1}$ \\
\hline & Young's modulus & $\mathrm{E}$ & $9.6 \times 10^{11}$ & $\mathrm{~Pa}$ \\
\hline & Poisson's ratio & $\sigma$ & 0.3 & \\
\hline & Electrical resistance/length at $293 \mathrm{~K}$ & $R_{L}$ & 23.0 & $\Omega \mathrm{m}^{-1}$ \\
\hline & Resistance temperature coefficient $=\left(1 / R_{0}\right)(d R / d T)$ & & $1.576 \times 10^{-4}$ & $\mathrm{~K}^{-1}$ \\
\hline \multirow[t]{9}{*}{ Air } & Temperature & $T_{\infty}$ & 293 & $\mathrm{~K}$ \\
\hline & Pressure & $p_{m}$ & 101.3 & $\mathrm{kPa}$ \\
\hline & Isobaric specific heat & $c_{p}$ & 1005 & $\mathrm{~J} \mathrm{~kg}^{-1} \mathrm{~K}^{-1}$ \\
\hline & Thermal conductivity & $\kappa$ & $2.57 \times 10^{-2}$ & 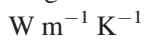 \\
\hline & Mass density & $\rho$ & 1.204 & $\mathrm{~kg} \mathrm{~m}^{-3}$ \\
\hline & Thermal diffusivity $=\kappa /\left(\rho c_{p}\right)$ & $\chi$ & $2.12 \times 10^{-5}$ & $\mathrm{~m}^{2} \mathrm{~s}^{-1}$ \\
\hline & Shear viscosity & $\mu$ & $1.81 \times 10^{-5}$ & $\mathrm{~Pa} \mathrm{~s}$ \\
\hline & Kinematic viscosity & $\nu$ & $1.51 \times 10^{-5}$ & $\mathrm{~m}^{2} \mathrm{~s}^{-1}$ \\
\hline & Prandtl number $=\nu / \chi$ & $\operatorname{Pr}$ & 0.708 & \\
\hline
\end{tabular}

TABLE III. Geometrical and thermophysical parameters of the $\mathrm{NiCr}$ wire and the surrounding air at atmospheric pressure and $T_{\infty}=293 \mathrm{~K} \cong 20{ }^{\circ} \mathrm{C}$. 
radian frequency to the quality factor of the fundamental resonance: $\eta=\omega_{\infty} / Q$.

The homogeneous oscillator equation (25) can be driven by the Lorentz force generated through the interaction of the alternating current, $i(t)$, and the magnetic induction, $B$, in the gap of width, $w$, between the magnet's pole pieces,

$$
\ddot{Z}+\eta \dot{Z}+\left(\frac{\pi c_{t}}{L}\right)^{2} Z=\frac{B w}{m_{s}} i(t) .
$$

The temperature-dependent resonance frequency of the wire vibrating in its fundamental mode, $\omega_{0}(\Delta T)$, as described by the simple harmonic oscillator of Eq. (25), can be expressed in terms of $\omega_{\infty}$ and the difference between the wire's mean temperature, $T_{\text {wire }}$, and the ambient air temperature far from the wire, $T_{\infty}: \Delta T=T_{\text {wire }}-T_{\infty}$,

$$
\omega_{0}(\Delta T)=\omega_{\infty}(1-\epsilon \Delta T)
$$

The frequency reduction coefficient, $\epsilon$, can be estimated from the experimental determination in Fig. 7 that shows a resonance frequency reduction with drive current of $-3.5 \mathrm{~Hz} /$ $\mathrm{A}_{a c}$. In Fig. 7, the resonance frequency is about $190 \pm 2 \mathrm{~Hz}$, so using Eqs. (9) and (10), and assuming $z_{p} \cong 3.0 \mathrm{~mm}$, thus making $v_{p}=2 \pi \times 190 \mathrm{~Hz} \times 0.003 \mathrm{~m}=3.6 \mathrm{~m} / \mathrm{s}$, implies that $\Delta T \cong 100 \mathrm{~K}$ for a current of one ampere. Differentiation of Eq. (27) provides the estimate for $\epsilon$,

$$
\epsilon=-\frac{1}{\omega_{\infty}} \frac{d \omega_{0}}{d T}=-\frac{2 \pi}{\omega_{\infty}} \frac{d f}{d i}\left(\frac{i}{\Delta T}\right) \cong 1.810^{-4} K^{-1} .
$$

\section{B. Convective transfer to surroundings}

As shown in Eq. (2), the Richardson number, $\mathrm{Ri} \cong 1$ $\times 10^{-4} \ll 1$, thus guaranteeing that for the vibration amplitudes of interest here, buoyancy-driven convective cooling can be ignored. For completeness, an equivalent buoyancydriven free convective cooling coefficient, $h_{\text {free }}$, can be extracted from the manufacturer's data for the $\mathrm{NiCr}$ wire's temperature as a function of current ${ }^{18}$ when combined with a steady-state energy balance equation for a stationary wire,

$$
C_{\text {wire }} d_{t} \Delta T=R_{d c} i^{2}-(\pi D L) h_{\text {free }} \Delta T .
$$

As before, $C_{\text {wire }}=c_{N i C r} \rho_{L} L \pi D^{2} / 4$, is the wire's heat capacity and $R_{d c}$ is the wire's electrical resistance. At steady state, a linear fit to the data supplied by the manufacturer results in $\Delta T(t \rightarrow \infty) \cong 300 i$. This relationship was independently confirmed by measuring the elongation of the wire using the LVDT and inferring the wire's temperature based on $\alpha_{\mathrm{NiCr}}=17 \mathrm{ppm} / \mathrm{K}$ for various electrical currents,

$$
h_{\text {free }}(\Delta T)=R_{d c}\left(\frac{\Delta T}{300}\right) \frac{1}{\pi D L \Delta T} \cong 0.326 \Delta T .
$$

By including $h_{\text {free }}$ in the heat transfer equation, the wire's temperature remains finite even in the absence of vibration.

The vibrational forced-convective cooling of the wire was calculated in Sec. IV by exploiting the Hilpert correlation of Eq. (4) for steady flow around an infinitely long cylinder ${ }^{30}$ to calculate a time-averaged effective heat transfer coefficient, $h_{\mathrm{eff}}$, by integrating over a wire that is vibrating in its fundamental half-wavelength mode using the Iguchi hypothesis, ${ }^{31}$ as shown in Fig. 4. Several alternative approaches were examined for pulsating flows ${ }^{39-42}$ as well as King's laws used for hot-wire anemometry ${ }^{43}$ that has been applied to oscillating air flow in the Rijke tube. ${ }^{44}$ All correlations produced similar results with a power-law dependence of the heat transport upon velocity, $v^{n}$, where $0.4 \leq n \leq 0.5$.

The Reynolds number used in Eq. (3) can now be replaced with a root-mean-squared value averaged over the wire's length, $\langle\overline{\operatorname{Re}}\rangle$,

$$
\langle\overline{\mathrm{Re}}\rangle=\frac{D}{L \nu}\left(\overline{\dot{Z}^{2}}\right)^{1 / 2} \int_{0}^{L} \sin (\pi x / L) \cdot d x=\frac{2 D}{\pi \nu}\left(\overline{\dot{Z}}^{2}\right)^{1 / 2} .
$$

With this definition, $1 \leq\langle\overline{\mathrm{Re}}\rangle \leq 62$, for our experimental conditions where $0.1 \mathrm{~mm}<Z<5.0 \mathrm{~mm}$ for frequencies around $190 \mathrm{~Hz}$. The averaged Nusselt number, $\langle\overline{\mathrm{Nu}}\rangle$, can be written in terms of this averaged Reynolds number as it was previously in Eq. (4),

$$
\langle\overline{\mathrm{Nu}}\rangle=0.911 \operatorname{Pr}^{1 / 3}\langle\overline{\mathrm{Re}}\rangle^{0.385} \text {. }
$$

Although it is not clear how to combine the free (buoyancy-driven) and forced convective heat transfer, they will just be added, since the free convection will be overwhelmed by forced convection if the wire is vibrating at our amplitudes of interest,

$$
\mathrm{Nu}=\mathrm{Nu}_{\text {free }}+\bar{C}\left(\overline{\dot{Z}^{2}}\right)^{n}
$$

From Eq. (32), $\bar{C}=0.911 \operatorname{Pr}^{1 / 3}\left[2 D /\left(\pi \nu_{f}\right)\right]^{0.385} \approx 1.99$ and $n=0.385 / 2$.

\section{Governing thermokinetic equations}

The dynamic effects contained in Eq. (26) and the thermal effects of Eqs. (29) and (33) can be combined into a set of two coupled nonlinear ordinary differential equations,

$$
\begin{aligned}
& \ddot{Z}+2 \xi \omega_{\infty} \dot{Z}+\omega_{\infty}^{2}[1-\epsilon \Delta T]^{2} Z=\frac{B w}{m_{s}} i_{p} \cos (\omega t) \\
& C_{\text {wire }} \Delta T=R_{d c} \frac{i_{p}^{2}}{2}-\pi D L\left[0.326 \Delta T+\frac{\kappa \bar{C}}{D}\left(\overline{\dot{Z}^{2}}\right)^{n}\right] \Delta T
\end{aligned}
$$

A new dimensionless damping factor, $\xi$, has been introduced to facilitate separation of the slow and fast time scales: $\eta=2 \xi \omega_{\infty} \Rightarrow 2 \xi=1 / Q$. The amplitude of the oscillatory current is $i_{p}$. These equations can be simplified by recognizing that the period of oscillations, $2 \pi / \omega_{\infty} \cong 0.005 \mathrm{~s}$, is much shorter than both the exponential time scales for the growth or decay of the resonance, $\tau=2 Q / \omega_{\infty}=\left(\xi \omega_{\infty}\right)^{-1}$, that appears in Eq. (34), or the wire's exponential thermal relaxation time, $\tau_{t h}$, calculated in Eq. (7), which controls the rateof-change of $\Delta T$ in Eq. (35). Both exponential time constants 
are on the order of one second. It is therefore possible to express the oscillatory amplitude, $Z(t)$, in terms of the driving frequency, $\omega$, to extract a slowly varying peak amplitude, $z_{p}$, and its phase, $\beta$, that are controlled by the slow variations in $\Delta T$,

$$
Z(t)=z_{p}(\xi t) \cos (\omega t+\beta(\xi t))
$$

\section{Separation by the method of multiple time scales}

The slow variations of the wire's amplitude, $z_{p}(\xi t)$, and the phase of that variation, $\beta(\xi t)$, as well as the slow variations in the temperature, can be described through the same set of coupled thermokinetic nonlinear differential equations that incorporate a solution of the form in Eq. (36),

$$
\begin{gathered}
\ddot{Z}+\omega_{\infty}^{2} Z=\xi\left[\alpha_{1} i_{p} \cos \omega t-2 \omega_{\infty} \dot{Z}+2 \frac{\epsilon}{\xi} \omega_{\infty}^{2} \Delta T Z\right. \\
\left.-\frac{\epsilon^{2}}{\xi} \omega_{\infty}^{2}(\Delta T)^{2} Z\right], \\
\dot{\Delta T}=\frac{1}{\tau_{t h}}\left[\alpha_{2} i_{p}^{2}-\left(\frac{\overline{\dot{Z}^{2}}}{v_{\text {ref }}^{2}}\right)^{n} \Delta T-\alpha_{3}(\Delta T)^{2}\right] .
\end{gathered}
$$

As in Eq. (7), a reference tranverse velocity $v_{\text {ref }}$ has been introduced to define the exponential thermal time constant, $\tau_{t h}$,

$$
\frac{1}{\tau_{t h}}=\frac{\pi L \lambda_{f} \bar{C}}{C_{\text {wire }}} v_{\text {ref }}^{2 n} .
$$

If we arbitrarily set the peak displacement amplitude equal to $1.0 \mathrm{~mm}$, then $v_{\text {ref }} \cong 0.84 \mathrm{~m} / \mathrm{s}$, so the thermal relaxation time becomes $\tau_{t h} \cong 1.3 \mathrm{~s}$. New constants are defined to properly scale the electrodynamic driving force, $\alpha_{1}=B w /\left(\xi m_{s}\right)$; the thermal relaxation time, $\alpha_{2}=R_{d c} \tau_{t h} /\left(2 C_{\text {wire }}\right)$; and the free convective (buoyancy-driven) cooling, $\alpha_{3}=\pi D L$ $\times 0.326 \tau_{t h}$. Following the approach of Nayfeh and Mook, ${ }^{37}$ we introduce two distinct time variables: The fast time scale is $t_{0}=t$ and the slow time scale is $t_{1}=\xi t$, where the dimensionless damping coefficient $\xi=2 Q^{-1} \ll 1$. The wire's displacement is assumed to depend upon both time scales, i.e., $Z(t)=Z\left(t_{0}, t_{1}\right)$, while the temperature difference depends only upon $t_{1}$. Under those circumstances, the derivatives of $Z$ and of $\Delta T$ with respect to time can be specified,

$$
\begin{aligned}
& \dot{Z}=\partial_{t_{0}} Z+\xi \partial_{t_{1}} Z, \\
& \ddot{Z}=\partial_{t_{0} t_{0}}^{2} Z+2 \xi \partial_{t_{1} t_{0}}^{2} Z+O\left(\xi^{2}\right), \\
& \dot{\Delta T}=\xi d_{t_{1}} \Delta T .
\end{aligned}
$$

Assuming that the forcing frequency is close to the natural frequency, the time-dependence of the source term, $\cos (\omega t)$, can be re-written,

$$
\cos \left(\omega_{\infty} t_{0}+\gamma t_{1}\right), \quad \text { with } \gamma=\left(\omega-\omega_{\infty}\right) / \xi .
$$

The solution can thus be found in terms of an order expansion in successive powers of $\xi$,

$$
Z\left(t_{0}, t_{1}\right)=Z_{0}\left(t_{0}, t_{1}\right)+\xi Z_{1}\left(t_{0}, t_{1}\right)+\cdots
$$

At the order $\xi^{0}$, the undamped simple harmonic oscillator of Eq. (25) is recovered,

$$
\partial_{t_{0} t_{0}}^{2} Z_{0}+\omega_{\infty}^{2} Z_{0}=0
$$

The well-known solution to Eq. (43) can be written as a sum of complex exponentials where $A^{*}$ is the complex conjugate of $A$,

$$
Z_{0}\left(t_{0}, t_{1}\right)=A\left(t_{1}\right) e^{j \omega_{\infty} t_{0}}+A^{*}\left(t_{1}\right) e^{-j \omega_{\infty} t_{0}}
$$

At order $\xi^{1}$, Eqs. (37) and (38) can be re-written in terms of both time scales for both dynamics and heat transfer,

$$
\begin{aligned}
& \partial_{t_{0} t_{0}}^{2} Z_{1}+\omega_{\infty}^{2} Z_{1}= \alpha_{1} i_{p} \cos \left(\omega_{\infty} t_{0}+\gamma t_{1}\right)-2 \omega_{\infty} \partial_{t_{0}} Z_{0} \\
&+ 2 \frac{\epsilon}{\xi} \omega_{\infty}^{2} \Delta T Z_{0}-\frac{\epsilon^{2}}{\xi} \omega_{\infty}^{2} \Delta T^{2} Z_{0} \\
&-2 \partial_{t_{0} t_{1}}^{2} Z_{0} \\
& \partial_{t_{1}} \Delta T=\frac{1}{\tau_{t h} \xi}\left[\alpha_{2} i_{p}^{2}-\left(\frac{\partial_{t_{0}}^{2} Z_{0}}{v_{r e f}^{2}}\right)^{n} \Delta T-\alpha_{3}(\Delta T)^{2}\right]
\end{aligned}
$$

Substitution of (44) into Eqs. (45) and (46) produces an ordinary nonlinear differential equation after terms corresponding to the homogeneous equation (43) have been eliminated,

$$
\begin{aligned}
-2 j \omega_{\infty}\left(d_{t_{1}} A+\omega_{\infty} A\right)+\frac{\epsilon}{\xi} \omega_{\infty}^{2} A \Delta T(2-\epsilon \Delta T) \\
+\frac{\alpha_{1} i_{p}}{2} e^{j \gamma t_{1}}=0
\end{aligned}
$$

where $A=a e^{j \varphi}$ is a complex valued, slowly varying function of $t_{1}=\xi t$, so Eq. (47) can be separated into its real and imaginary parts,

$$
\begin{aligned}
& -2 \omega_{\infty}\left(d_{t_{1}} a+\omega_{\infty} a\right)+\frac{\alpha_{1} i_{p}}{2} \sin \left(\gamma t_{1}-\varphi\right)=0 \\
& 2 a \omega_{\infty} d_{t_{1}} \varphi+\frac{\epsilon}{\xi} \omega_{\infty}^{2} a \Delta T(2-\epsilon \Delta T) \\
& +\frac{\alpha_{1} i_{p}}{2} \cos \left(\gamma t_{1}-\varphi\right)=0
\end{aligned}
$$

A final resulting set of three ordinary coupled nonlinear differential equations can be written that will be suitable for numerical solution and will dictate the slow time evolution of the amplitude, the phase of the slow modulation, and the temperature difference where $t_{1}=\xi t$,

$$
\begin{aligned}
& d_{t_{1}} a=\frac{\alpha_{1} i_{p}}{4 \omega_{\infty}} \sin \left(\gamma t_{1}-\varphi\right)-\omega_{\infty} a, \\
& d_{t_{1}} \varphi=-\frac{\alpha_{1} i_{p}}{4 a \omega_{\infty}} \cos \left(\gamma t_{1}-\varphi\right)-\frac{\epsilon}{2 \xi} \omega_{\infty} \Delta T(2-\epsilon \Delta T), \\
& d_{t_{1}} \Delta T=\frac{1}{\tau_{t h} \xi}\left[\alpha_{2} i_{p}^{2}-\left(\frac{2 \omega_{\infty}^{2} a^{2}}{v_{r e f}^{2}}\right)^{n} \Delta T-\alpha_{3}(\Delta T)^{2}\right] .
\end{aligned}
$$


This system of coupled equations can be solved numerically using a Runge-Kutta scheme to produce the time evolution of $Z(t)$, where $z_{p}=2 a$ and $\beta=\varphi-\left(\omega-\omega_{\infty}\right) t$,

$$
\begin{aligned}
Z(t) & =2 a(\xi t) \cos \left(\omega_{\infty} t+\varphi(\xi t)\right) \\
& =z_{p}(\xi t) \cos (\omega t+\beta(\xi t)) .
\end{aligned}
$$

\section{SAMPLE OF THE NUMERICAL SIMULATIONS}

In this section, numerical solutions to the set of equations derived previously that were intended to describe the slowly varying amplitude and temperature of the wire near its resonance frequency will be solved numerically and graphical summaries of those solutions will be presented and discussed. Those results are intended to demonstrate that our analysis reproduces all the new experimentally observed behaviors. This agreement gives us confidence that those equations can be exercised to fully examine the parameter space under which the periodic amplitude modulations can be observed.

\section{A. Periodic amplitude modulation}

The most striking experimental result was the observation of the spontaneous and stable amplitude modulation exhibited by all of our "burning wire" apparatuses listed in Table I. Figure 12 shows a large modulation when the driven frequency is $\omega=0.985 \omega_{\infty}$ and no modulation if the drive frequency is only slightly farther from resonance at $\omega=0.98 \omega_{\infty}$.

Figure 12 reveals several interesting features. First, if the drive current's frequency is tuned farther from resonance than is necessary to excite the amplitude modulations, the resultant constant amplitude is significantly less than the modulated amplitude when the wire is driven at a slightly higher frequency. Its temperature is both higher and stable. This is not surprising since the lower amplitude is expected from the shape of the resonance curve and the higher temperature is a consequence of less forced convective cooling corresponding to the wire's lower oscillatory velocity.

Driven closer to the resonance frequency, it is clear that the modulation of the amplitude is large, with a modulation depth of about $86 \%$. By examining the expanded views once the modulation has reached its steady-state condition, at the right of Fig. 12, it is possible to observe the phase shift between the amplitude variations and the temperature variations that indicate the retarded response of the amplitude to changes in the temperature caused by the amplitude modulations. Focusing on the amplitude minimum near $t=86 \mathrm{~s}$, the corresponding slope of the temperature difference has its greatest positive value. The temperature is rising and the resonance frequency, $\omega_{0}(\Delta T)$ in Eq. (27), is being reduced, thus bringing the drive frequency closer to the resonance frequency and leading to the subsequent increase in the amplitude of the wire's vibration.

Similarly, at the amplitude maximum, the slope of the temperature difference has its largest negative value so the resonance frequency, $\omega_{0}(\Delta T)$, is increasing, thereby detuning the resonance and reducing the wire's subsequent vibrational amplitude.

The results of the calculations shown in Fig. 13 demonstrate that the mathematical model also exhibits the increase in the modulation period, $T_{\text {mod }}$, as the tuning ratio, $\omega / \omega_{\infty}$, increases as was observed experimentally and shown in Fig. 3. In Fig. 13, five different drive currents were used with $0.2 A \leq i_{p} \leq 1.0 \mathrm{~A}$. The higher currents correspond to higher heating powers. At the highest three values of drive current, the frequency range of modulation behavior increases with increasing current. The two lowest currents exhibit no amplitude modulation at all.

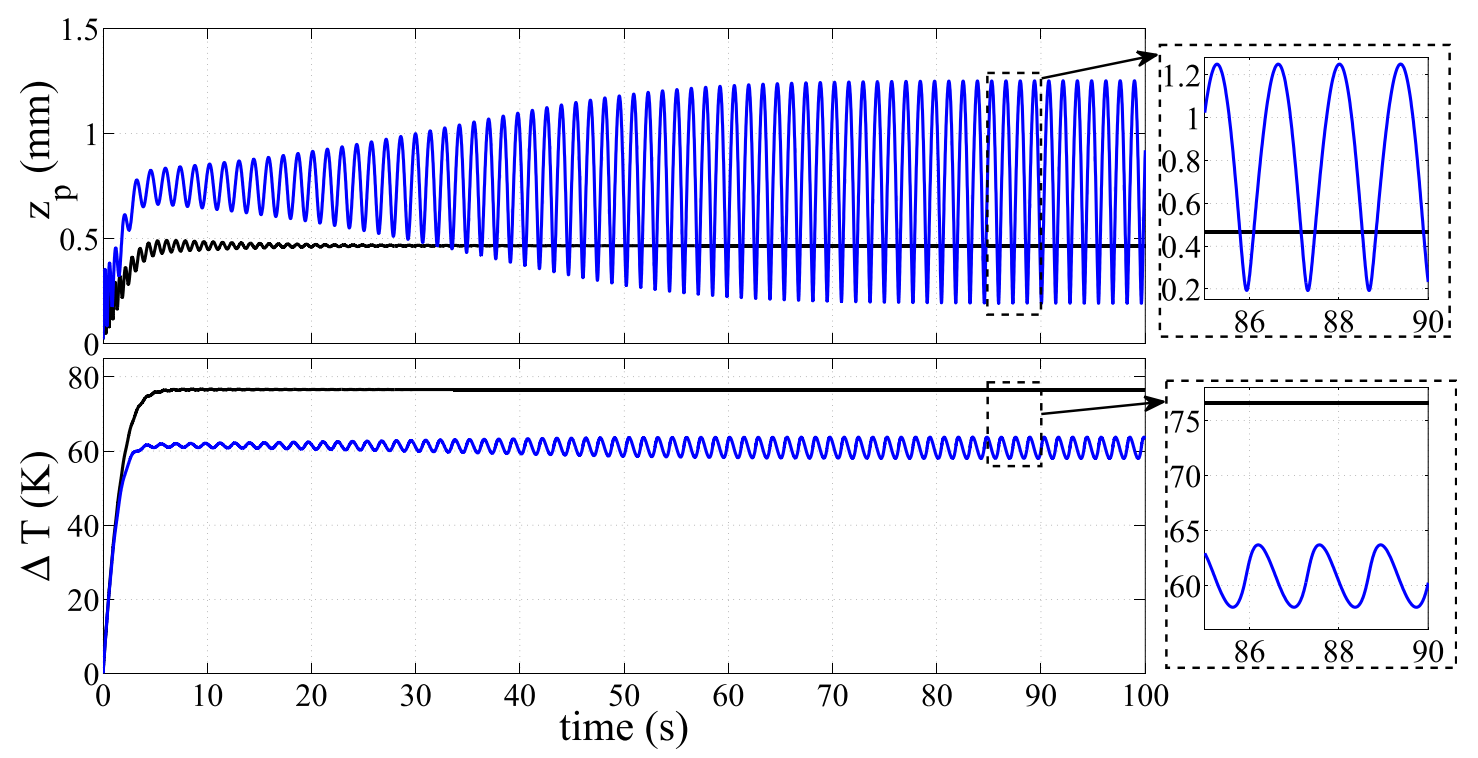

FIG. 12. (Color online) Two examples of the calculated transient regimes leading to either the steady-state amplitude modulations (blue lines), with $T_{\text {mod }}=1.37 \mathrm{~s}$, or to smaller constant-amplitude oscillations (black lines). The upper graph represents the peak amplitude, $z_{p}$, as a function of time and the lower graph represents the temperature difference, $\Delta T$, between the wire and the temperature, $T_{\infty}$, of the surrounding air far from the wire. Both transient regimes correspond to an alternating current with amplitude $i_{p}=0.8 \mathrm{~A}$, that passes through a NiCr wire of length $L=0.5 \mathrm{~m}$. In both cases, the initial amplitude, $z_{p}(t=0)=0.1 \mathrm{~mm}$ and $\Delta T(t=0)=0 \mathrm{~K}$. The drive frequency for the black curve is $\omega=0.98 \omega_{\infty}$. For the blue curve, $\omega=0.985 \omega_{\infty}$. 

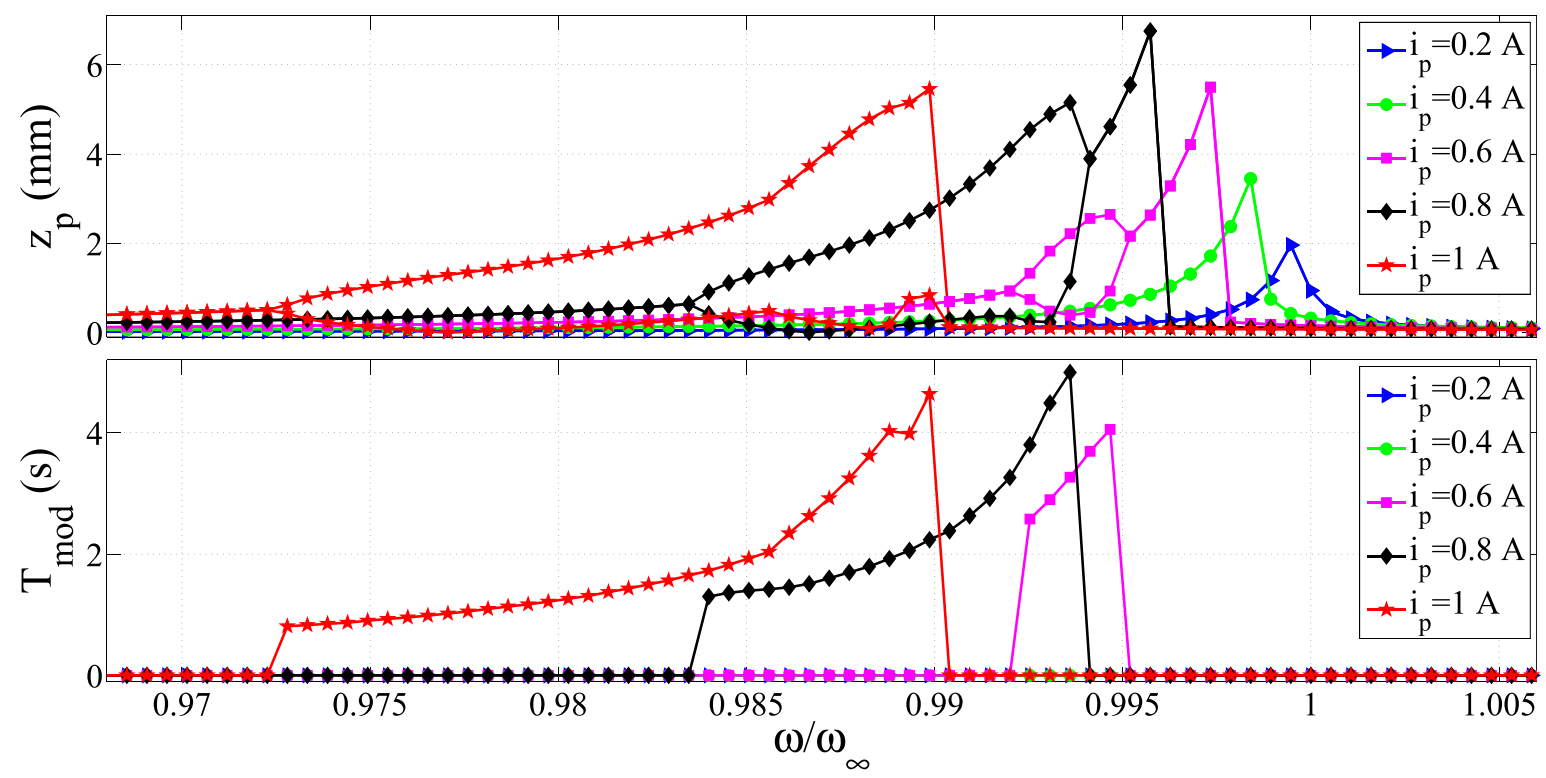

FIG. 13. (Color online) The amplitude, $z_{p}$, of the wire's oscillations and the period of those modulations, $T_{\text {mod }}$, are plotted as a function of the tuning ratio, $\omega / \omega_{\infty}$, as the drive frequency is increased from $\omega=0.96 \omega_{\infty}$ to $1.04 \omega_{\infty}$ for five different drive current amplitudes, $0.2 \mathrm{~A} \leq i_{p} \leq 1.0 \mathrm{~A}$. In the upper graph, amplitude modulation is indicated by the appearance of two amplitude values for each frequency. The modulation period is shown on the lower graph.

\section{B. Thermokinetic coupling}

It is the interaction of the wire's dynamics and its heat transfer to the surrounding fluid that makes this problem interesting. The amplitude of the wire's motion depends upon the tuning ratio, $\omega / \omega_{\infty}$, which influences its temperature. The changes in the wire's temperature change the tuning ratio. Both of those effects are retarded by exponential times related to the quality factor of the resonance and to the thermal equilibration rate of the wire. Figure 14 demonstrates that the correlation chosen for calculation of the convective heat transfer coefficient is not particularly important, but if heating is absent, as it is for a copper or aluminum wire, there is no amplitude modulation or hysteretic behavior.

The black and blue data in Fig. 14 differ only by the inclusion or omission of the heat transfer by free convection. Those results are indistinguishable, as expected based on the very small value of the Richardson number at typical values of vibration amplitude. The forced-convective heat transport for both black and blue is based on the Nusselt number correlation that was the result of a numerical study by Su et al., ${ }^{40}$

$$
\langle\overline{\mathrm{Nu}}\rangle=0.624\langle\overline{\mathrm{Re}}\rangle^{0.475}
$$

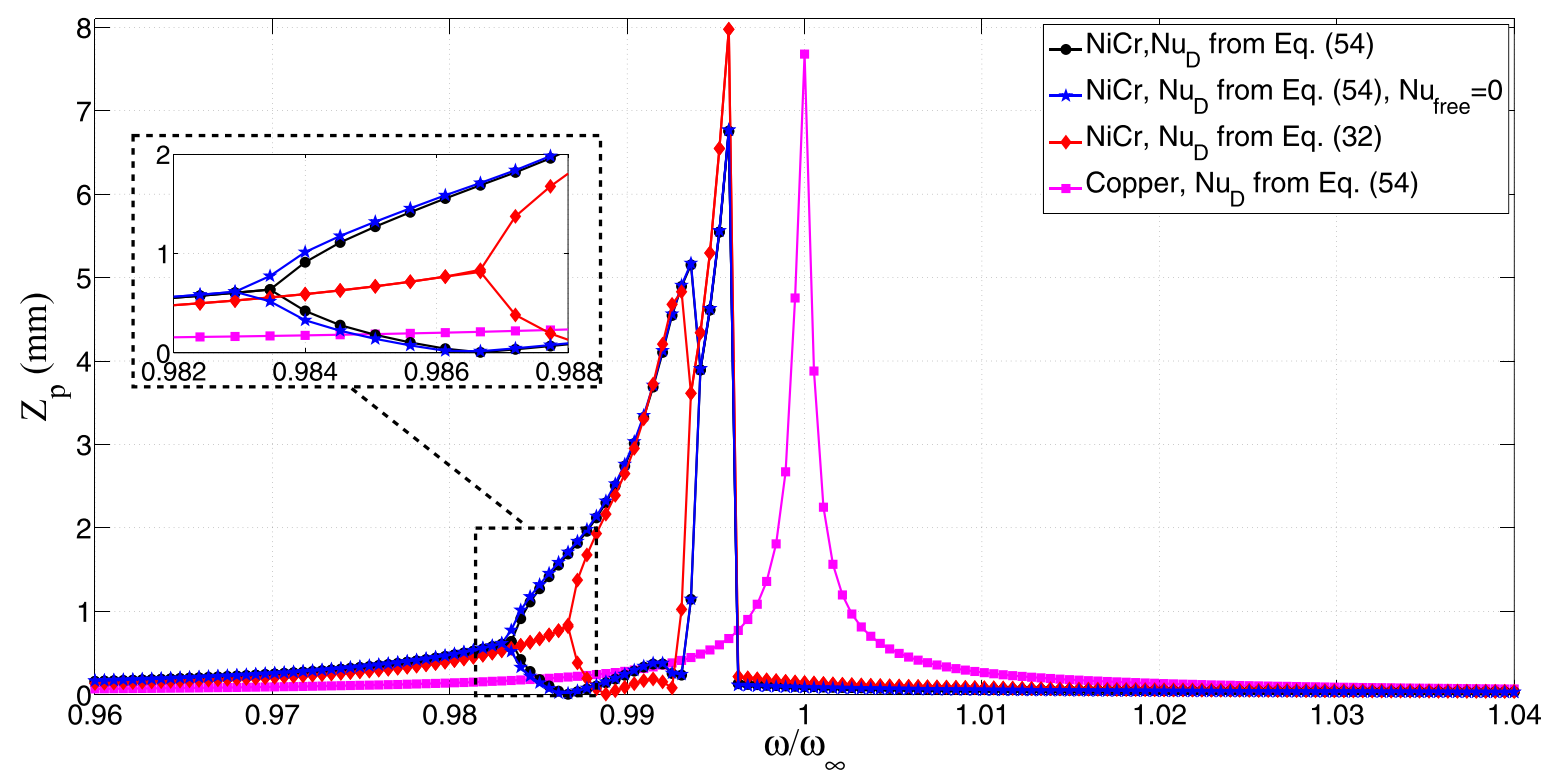

FIG. 14. (Color online) The amplitude, $z_{p}$, of the wire's vibrations is shown as a function of the tuning ratio, $\omega / \omega_{\infty}$, as the drive frequency is increased from $\omega=0.96 \omega_{\infty}$ to $1.04 \omega_{\infty}$ for $i_{p}=1.0 \mathrm{~A}$ and $L=0.5 \mathrm{~m}$. Amplitude modulation is indicated by the presence of two data points for each value of tuning ratio where the higher and lower points correspond to the maximum and minimum amplitudes. The blue and black points are nearly indistinguishable since they differ only by inclusion of the free convection contribution for the black points, as shown in Eq. (33). Both black and blue curves use the Nusselt number correlation provided in Eq. (54) that depends upon $\langle\overline{\operatorname{Re}}\rangle^{0.475}$. The red curve uses the correlation of Eq. (32) that depends upon $\langle\overline{\mathrm{Re}}\rangle^{0.385}$. Unlike the other results in this figure, which were calculated for NiCr wire, the pink curve was calculated for copper wire and shows no amplitude modulation. 

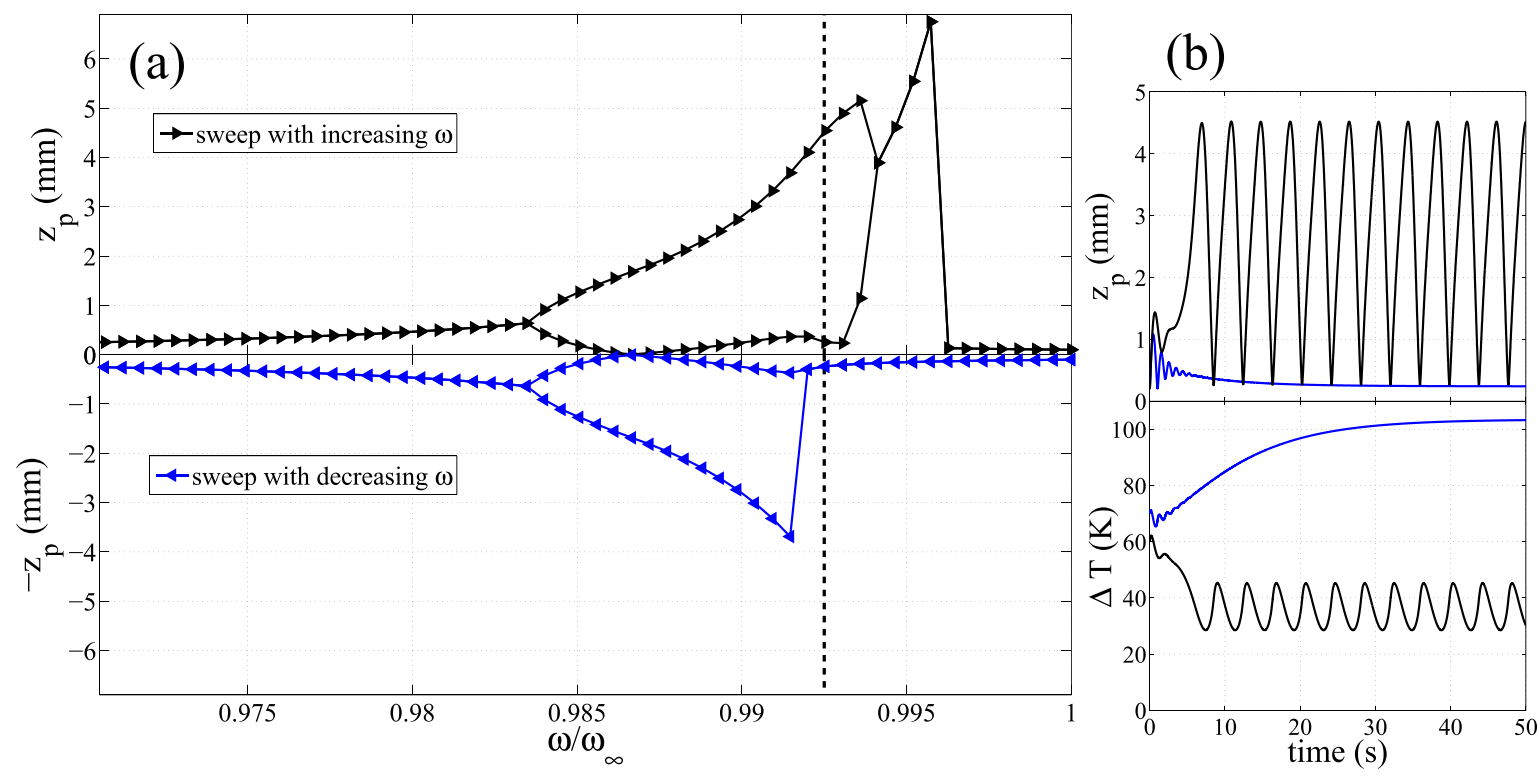

FIG. 15. (Color online) (a) The amplitudes of the wire's oscillations, $z_{p}$, are shown as a function of the drive ratio, $\omega / \omega_{\infty}$, either with $\omega$ increasing (upper graph, black curve) or decreasing (lower graph, blue curve) for a drive current, $i_{p}=1.0 \mathrm{~A}$. (b) The upper and lower graphs show the transient evolution of $z_{p}$ and $\Delta T$ for $i_{p}=1.0 \mathrm{~A}$ and $\omega / \omega_{\infty}=0.9925$. That frequency ratio is represented by the dashed vertical line in (a). Both of those two transient regimes are obtained from an initial displacement of $z_{p}(t=0)=1.0 \mathrm{~mm}$, but the assigned initial temperature difference, $\Delta T(t=0)$, is either $70 \mathrm{~K}$ (blue curve) or $60 \mathrm{~K}$ (black curve)

The red points in Fig. 14 use the result of Eq. (32) that has a power-law dependence on Reynolds number that is 0.385 instead of 0.475 . Although there are small differences between the black and red data, the basic modulation behavior is fairly insensitive to the choice of Reynolds number exponent over this range.

The pink data in Fig. 14 are produced by the solution of the same equations but using copper wire which produces insignificant heating and therefore does not exhibit any thermokinetic effects.

\section{Tuning hysteresis}

As mentioned at the beginning of Sec. III, the wire's vibrational amplitude increases as the resonance frequency is approached from below, but when the resonance frequency is exceeded, the amplitude drops precipitously. If the frequency is reduced from above the resonance frequency, the amplitude does not start increasing again until the frequency is well below that resonance peak frequency that was achieved when the frequency was increasing prior to collapse. This hysteretic behavior is also evident from the tuning curve in Fig. 2.

The mathematical model clearly exhibits this hysteretic behavior as illustrated in Fig. 15. As the frequency is increased, the vibrational amplitude, $z_{p}$, increases slowly until the amplitude modulation manifests above about $\omega / \omega_{\infty} \cong 0.9837$. Further frequency increases lead to a more rapid growth in peak amplitude along with deep amplitude modulation. As the frequency is increased, the modulation ceases but the amplitude increases just before the resonance collapses. Further frequency increases are not accompanied by any vibration.

Decreasing the drive frequency after collapse does not restore the modulation until the frequency is well below the frequency were modulations were large while the frequency was being increased.

The dashed vertical line in Fig. 15(a) that is drawn at $\omega / \omega_{\infty}=0.9925$ marks the frequency value that exhibited substantial modulation when the frequency was being increased and a very small vibrational amplitude when the frequency was being decreased. The transient behavior at that frequency ratio is shown in Fig. 15(b). For the case where the frequency is being swept upward, the time evolution is exactly like that shown in Fig. 12 that led to stable amplitude modulation. The downward swept frequency at that point shows no growth and no stable steady-state modulation but simply decays to a very small vibrational amplitude while $\Delta T$ increases monotonically toward a constant value that is higher due to the lack of forced convective cooling.

\section{CONCLUSIONS}

We consider ourselves to be fortunate to have produced a "burning wire" demonstration device that exposed us to a portion of parameter space that exhibited the unanticipated periodic modulation of the resonant wire's vibrational amplitude. This paper has described the experimental investigations that led to an appreciation of the thermokinetic coupling between the wire's motion and its temperature due to convective cooling and to the changes in the wire's resonance frequency that are influenced by those temperature changes, causing the tensioning mass to do work against the force of gravity. Imposition of the Boltzmann-Ehrenfest adiabatic principle provided the necessary connection between that thermally induced mechanical work and the change in the normal mode frequency of the wire's half-wavelength resonance.

At this point, further investigations will be required to make a quantitative connection between the temperaturefrequency shifts that are a consequence of adiabatic invariance. 
The results obtained experimentally are consistent with the theoretical ones based on the coupled thermokinetic equations (34) and (35), but further experimental work would be required so as to provide a mapping of parameter space that exhibits stable periodic amplitude modulations.

\section{ACKNOWLEDGMENTS}

This research was supported by the HUB Acoustic Program at Le Mans Université. Professor Adrian Pelat provided access to the infrared video camera and his summer intern, Gildas Cozanet, provided assistance making the twenty-four videos and also provided instruction in the use of the camera's post-processing software.

\section{APPENDIX: SOME SIMPLE EXAMPLES OF ADIABATIC INVARIANCE}

When Lord Rayleigh introduced his Reciprocity Principle in 1883, he felt it was necessary to give "a few examples [to] promote the comprehension of a theorem which, on account of its extreme generality, may appear vague." ${ }^{45}$ We believe that the same argument could be applied to the principle of adiabatic invariance. ${ }^{34}$ In this case, a simple example that is relevant to the change in resonance frequency of the vibrating wire is the simple pendulum. If the point of attachment at the top of the pendulum is treated as being the same as the two ball-bearing races used in the third apparatus, those rollers will allow the pendulum to swing at its natural frequency and also allow the length of the pendulum, $\ell$, to be increased or decreased while the pendulum is swinging. ${ }^{46}$

Adiabatic invariance is a classical mechanical concept ${ }^{47}$ that is more familiar today in its quantum mechanical manifestation where the "constant" is universal for atomic systems: $E / f=h$, and $h$ is Planck's constant. For the classical result, "adiabatic" implies that the energy changes happen sufficiently slowly that they do not alter the vibrational mode shape. This is clearly the case for our vibrating wire since it always remains in its fundamental half-wavelength mode. It is also true for the swinging pendulum. In both cases, the time scale for the vibration is much shorter than the time scale for changes in amplitude (determined by the quality factor of the resonance) or changes in temperature, that occur on a time scale, $\tau_{\text {th }} \cong 1 \mathrm{~s} \gg \omega^{-1} \cong 10^{-3} \mathrm{~s}$, as calculated in Eq. (7).

The oscillation frequency of a pendulum, $f_{0}$, can easily be calculated from the length of the pendulum and the gravitational acceleration: $f_{0}=(1 / 2 \pi)(g / \ell)^{1 / 2}$. If the pendulum is shortened while it is swinging, its frequency increases. Another way to understand this frequency increase is to calculate the work done against the centripetal acceleration of the mass that is produced by shortening the string. The ratio of that work to the pendulum's kinetic energy will be the same as the ratio of the change in frequency, $\delta f$, produced by the shortening, to the initial frequency, $f_{0}$, of the pendulum before the string was shortened,

$$
\left(\frac{E}{f}\right)_{a d}=\text { constant } \Rightarrow \delta\left(\frac{E}{\omega}\right)=0 \quad \text { or } \frac{\delta E}{E}=\frac{\delta \omega}{\omega} .
$$

When the pendulum is shortened, the pendulum is doing work against the centripetal force, thus $\delta E>0$, and the frequency increases, so $\delta f>0$. That work also causes the angular displacement of the pendulum's motion to increase. If the pendulum's string is allowed to lengthen, the centripetal force is doing work on the pendulum and $\delta E<0$ resulting in a decrease in the pendulum's frequency, $\delta f<0$, and a decrease in the angular amplitude of oscillations.

The bending of a guitar note is another simple example that can be easily demonstrated with the resulting increase in pitch calculable using adiabatic invariance. With a finger depressing a string to make contact with a fret, the frequency of the note produced when the string is plucked is independent of the amplitude of vibration for small vibrational amplitudes. If the finger is moved perpendicular to the string along the fret while the string is vibrating, an amount of work, $W$, is done against the perpendicular component of the string's tension, $\mathcal{T}_{\perp}$, to increase the tension of the string thus also increasing the pitch of the note,

$$
W=\int_{0}^{y} \mathcal{T}_{\perp} d y=\int_{0}^{y} \mathcal{T} \sin \theta d y \cong \int_{0}^{y} \mathcal{T} \frac{y}{x} \cdot d y=\mathcal{T} \frac{y^{2}}{2 x} .
$$

Since the mode shape of the string does not change, and adiabatic invariance requires that $E / f=$ constant, the increase in frequency, $\delta f$, is related to the work done to "bend" the note, $W$,

$$
\frac{E}{f}=\frac{E+W}{f+\delta f}=\text { constant } \Rightarrow \frac{\delta f}{f}=\frac{W}{E} .
$$

The energy stored by the string's tension, $E$, can be calculated by integrating the tension which is zero for the limp string, over the length change, $\Delta L$, produced by the tensioning pegs used to tune the guitar.

${ }^{1}$ P. M. Morse and K. U. Ingard, Theoretical Acoustics (McGraw-Hill, New York, 1968), see Sec. 14.3.

${ }^{2}$ M. S. Korman and T. McClanahan, "Standing waves on an electrically heated wire, revisited: Demonstration of glow at the nodes," J. Acoust. Soc. Am. 137(4), 2412 (2015).

${ }^{3}$ P. Gluck, "Hot-wire vibrations get waves standing," Phys. Ed. 40(4), 315-316 (2005).

${ }^{4}$ G. Kirchhoff, Vorlesungen uber Mathematische Physik (B. G. Teubner, Leipzig, 1883).

${ }^{5}$ J. W. Strutt (Lord Rayleigh), The Theory of Sound, 2nd ed. (Dover, New York, 1945).

${ }^{6} \mathrm{~J}$. Johnson and A. Bajaj, "Amplitude modulated and chaotic dynamics in resonant motion of strings,” J. Sound Vib. 128(1), 87-107 (1989).

${ }^{7}$ N. B. Tufillaro, "Nonlinear and chaotic string vibrations," Am. J. Phys. 57(5), 408-414 (1989).

${ }^{8}$ L. E. Kinsler, A. R. Frey, A. B. Coppens, and J. V. Sanders, Fundamentals of Acoustics, 4th ed. (Wiley, New York, 2000).

${ }^{9}$ P. M. Morse, Vibration and Sound, 2nd ed. (Acoustical Society of America, New York, 1995).

${ }^{10}$ L. L. Beranek, Acoustics (Acoustical Society of America, New York, 1996).

${ }^{11}$ D. T. Blackstock, Fundamentals of Physical Acoustics (Wiley, New York, 2000).

${ }^{12}$ A. D. Pierce, Acoustics: An Introduction to its Principles and Applications (McGraw-Hill, New York, 1981).

${ }^{13}$ E. Skudrzyk, The Foundations of Acoustics (Springer, Berlin, 1971).

${ }^{14} \mathrm{~S}$. Temkin, Elements of Acoustics (Wiley, New York, 1981). 
${ }^{15}$ J. H. Ginsberg, Acoustics-A Textbook for Engineers and Physicists (ASA Press-Springer, New York, 2018).

${ }^{16}$ Eclipse Magnetics, Model E803.

${ }^{17}$ Omega Engineering, Model LCL-010 Full-Bridge Thin-Beam Load Cell with LCM-CL1 Mount, Stamford, CT 06907.

${ }^{18}$ Omega Engineering, NiCr resistance wire, Model No. NI60-010, Stamford, CT 06907.

${ }^{19}$ Peanut oil has the highest "smoke temperature" of the cooking oils commonly available in supermarkets.

${ }^{20}$ TE Connectivity Sensor Solutions, E-300 Series Economy Series AC LVDT, S/N 0901, 1000 Lucas Way, Hampton, VA 23666.

${ }^{21}$ J. Fraden, AIP Handbook of Modern Sensors: Physics, Designs, and Applications (American Institute of Physics, New York, 1993).

${ }^{22}$ Polytec OFV-5000, sensor head OFV-505, S/N: 0158704, Irvine, CA 92618.

${ }^{23}$ McMaster-Carr, Part No. 60355K501, R2 Ball Bearing (1/2 in. shaft, $3 / 8$ in. OD), Chicago, IL 60680.

${ }^{24}$ Analog Devices AD 620 Instrumentation Amplifier, Norwood, MA 02062.

${ }^{25}$ FLIR Systems, Inc., Model X6580 SC.

${ }^{26} \mathrm{~J}$. Liu and S. Garrett, "Characterization of a small moving-magnet electrodynamic linear motor," J. Acoust. Soc. Am. 118(4), 2289-2294 (2005).

${ }^{27}$ A. B. Pippard, The Physics of Vibration (Cambridge, New York, 1989), see Chap. 9.

${ }^{28}$ The idea of superimposing a steady flow on the heated vibrating wire was first suggested by Prof. Dr. Ir. A. (Mico) Hirschberg, retired from the Technische Universiteit-Eindhoven.

${ }^{29}$ J. Turner, Buoyancy Effects in Fluids (Cambridge University Press, New York, 1973).

${ }^{30}$ F. P. Incropera and D. P. DeWitt, Fundamentals of Heat and Mass Transfer, 4th ed. (Wiley, New York, 1996).

${ }^{31}$ M. Iguchi, M. Ohmi, and K. Meagawa, "Analysis of free oscillating flow in a u-shaped tube,” Bull. JSME 25, 1398-1405 (1982).

${ }^{32}$ Another way to appreciate this limit is to calculate the thermal time constant, $\tau_{\text {wire }}$, for radial equilibration of the wire's temperature: $\tau_{\text {wire }}=\rho_{N i C l} c_{N i C r} D^{2} /\left(8 \kappa_{N i C r}\right) \cong 2.56 \times 10^{-3} \mathrm{~s} \ll \tau_{\text {th }} \cong 0.9 \mathrm{~s}$.

${ }^{33}$ Jelt Noir Mat heat-resistant paint. This paint remains stable up to temperatures of $700{ }^{\circ} \mathrm{C}$.
${ }^{34} \mathrm{M}$. Greenspan, "Simple derivation of the Boltzmann-Ehrenfest adiabatic principle," J. Acoust. Soc. Am. 27(1), 34-35 (1955).

${ }^{35} \mathrm{H}$. Gottlieb, "Non-linear vibration of a constant-tension string," J. Sound Vib. 143(3), 455-460 (1990).

${ }^{36}$ H. M. Ledbetter and R. P. Reed, "Elastic properties of metals and alloys, I. Iron, nickel, and iron-nickel," J. Phys. Chem. Ref. Data 2(3), 531-617 (1973).

${ }^{37}$ A. H. Nayfeh and D. T. Mook, Nonlinear Oscillations (Wiley, New York, 1979).

${ }^{38}$ C. Gough, "The Non-linear free-vibration of a damped elastic string," J. Acoust. Soc. Am. 75(6), 1770-1776 (1984).

${ }^{39}$ H. Iwai, T. Mambo, N. Yamamoto, and K. Suzuki, "Laminar convective heat transfer from a circular cylinder exposed to a low frequency zeromean velocity oscillating flow," Int. J. Heat Mass Transf. 47(21), 4659-4672 (2004).

${ }^{40}$ Y. Su, J. Davidson, and F. Kulacki, "Numerical study on mixed convection from a constant wall temperature circular cylinder in zero-mean velocity oscillating cooling flows," Int. J. Heat Fluid Flow 44, 95-107 (2013).

${ }^{41}$ H. Park and M. Gharib, "Experimental study of heat convection from stationary and oscillating circular cylinder in cross flow," Trans. ASME J. Heat Transf. 123(1), 51-62 (2001).

${ }^{42}$ C. Cheng, H. Chen, and W. Aung, "Experimental study of the effect of transverse oscillation on convection heat transfer from a circular cylinder," Trans. ASME J. Heat Transf. 119(3), 474-482 (1997).

${ }^{43}$ L. King, "On the convection of heat from small cylinders in a stream of fluid: Determination of the convection constants of small platinum wires, with applications to hot-wire anemometry," Proc. R. Soc. London A 90(622), 563-570 (1914).

${ }^{44}$ M. Heckl, "Nonlinear acoustic effects in the Rijke tube," Acustica 72(1), 63-71 (1990).

${ }^{45}$ J. W. Strutt (Lord Rayleigh), Scientific Papers (Dover, New York, 1964), Vol. I.

${ }^{46}$ S. L. Garrett, Understanding Acoustics: An Experimentalist's View of Sound and Vibration (ASA Press-Springer, New York, 2017).

${ }^{47}$ L. D. Landau and E. M. Lifshitz, Mechanics (Pergamon Press, New York, 1959), see Sec. 49 where the simple harmonic oscillator is used as an example of the general derivation based on Hamiltonian mechanics. 